\title{
基于实验室模拟研究二次有机气溶胶生成及老化机制
}

\author{
王辉 ${ }^{a}$ 俞颖 ${ }^{a}$ 唐荣志 ${ }^{a}$ 郭松*,,$b$ \\ ( ${ }^{a}$ 北京大学环境模拟与污染控制国家重点实验室 北京大学环境科学与工程学院 北京 100871) \\ ( ${ }^{b}$ 江苏省大气环境与装备技术协同创新中心 南京信息工程大学 南京 210044)
}

\begin{abstract}
摘要 由于二次有机气溶胶(SOA)对气候变化、区域污染和人体健康具有明显的影响, 因此受到了广泛的关注. 基于实 验室的方法可以在稳定可控的条件下探讨 SOA 的生成机制, 其中环境烟雾箱和气溶胶生成潜势反应器是最常用的两 种模拟工具. 本文综述了基于这两种模拟工具对 SOA 产率的影响因素、SOA 生成机制和 SOA 老化过程中性质演变特 征的研究. 影响 $\mathrm{SOA}$ 产率的因素主要包括 $\mathrm{OH}$ 暴露量, $\mathrm{NO}_{x}$ 水平 $\left(\mathrm{VOCs} / \mathrm{NO}_{x}\right)$, 种子颗粒物的浓度及种子颗粒物的化学 组成. $\mathrm{SOA}$ 产率随着 $\mathrm{OH}$ 暴露量、 $\mathrm{VOCs} / \mathrm{NO}_{x}$ 比值的增加均先增后减; 种子颗粒物的存在会通过提高气态中间产物的凝 结汇，从而促进物质从气相到颗粒相的转化; 酸性种子颗粒物可以通过提高摄取系数并提供酸催化条件促进 SOA 的 生成; 种子颗粒物中的金属离子和矿物质也会通过催化作用或者影响氧化剂的产生等过程对 SOA 的生成和老化产生 作用. 本综述还总结了不同源排放气态前体物 SOA 的生成潜势以及生成 SOA 的特征. 等效光氧化龄约为 $2 \sim 3 \mathrm{~d}$ 时, 汽 油发动机排放生成 SOA 量达到最高值, 增长倍数(SOA/POA, POA 即为一次有机气溶胶)约为 10 14, SOA 生成潜势约 为 $400 \sim 500 \mathrm{mg} / \mathrm{kg}$ fuel; 生物质燃烧排放, 在等效光氧化龄约为 $3 \sim 4 \mathrm{~d}$ 时, SOA 增长倍数最大, 平均约为 1.42 7.6; 而 其他源如天然气燃烧、餐饮等排放也具有很高的 SOA 生成潜势, 天然气燃烧排放后 SOA 的增长倍数高达 268 倍, 餐 饮源排放 SOA 的增长倍数约为 $3 \sim 8$ 倍. 不同地区的实际大气中气态前体物氧化生成 SOA 的最高值出现在等效光化学 龄为 $2 \sim 4 \mathrm{~d}$ 时. 综合不同研究中源排放和实际大气中前体物生成 SOA 演化特征发现, 随着 OH 暴露量的增加, SOA 的 氧化态逐渐增加, $\mathrm{O} / \mathrm{C}$ 比约从 0.2 增长到 $1.3, \mathrm{O} / \mathrm{C}$ 与 $\mathrm{H} / \mathrm{C}$ 变化斜率均在 -1 到 0 之间, 说明氧化机制可能包括生成差基、 过氧着基以及羧酸基团的物质; 氧化过程中 SOA 的挥发性逐渐降低, 吸湿性逐渐增加. SOA 生成过程中间态物种的测 量技术开发、复杂体系下 SOA 生成机制的研究和 SOA 演化过程中特征的表征是未来 SOA 研究的重要方面. 关键词 二次有机气溶胶; 产率; 生成潜势; 烟雾箱; 氧化流动管
\end{abstract}

\section{Research on Formation and Aging of Secondary Organic Aerosol Based on Simulation Methods}

\author{
Wang, Hui $^{a} \quad$ Yu, Ying $^{a} \quad$ Tang, Rongzhi ${ }^{a} \quad$ Guo, Song ${ }^{*, a, b}$ \\ ( ${ }^{a}$ State Key Joint Laboratory of Environmental Simulation and Pollution Control, \\ College of Environmental Sciences and Engineering, Peking University, Beijing 100871) \\ $\left({ }^{b}\right.$ Cooperative Innovation Center of Atmospheric Environment and Equipment Technology, \\ Nanjing University of Information Engineering, Jiangsu, Nanjing 210044)
}

\begin{abstract}
Secondary organic aerosol (SOA) is a major component of aerosols in the atmosphere, which plays a crucial role in climate change, regional pollution and human health. Laboratory simulations are usually used to mimic SOA formation. The most commonly used simulation facilities are environmental chambers and potential aerosol mass (PAM) reactors. Here in this work, we review the studies about influencing factors and mechanisms of SOA formation, as well as the evolution of SOA aging. We summarize the influencing factors on $\mathrm{SOA}$ yields, i.e. $\mathrm{OH}$ exposure, $\mathrm{NO}_{x}$ level, and the loading and chemical composition of seed particles. The effects of $\mathrm{NO}_{x}$ level (i.e. $\mathrm{VOCs} / \mathrm{NO}_{x}$ ) and $\mathrm{OH}$ exposure are nonmonotonic. The $\mathrm{NO}_{x}$ level influences the fate of $\mathrm{RO}_{2}$ radicals, so SOA yields will increase and then decrease with the addition of $\mathrm{NO}_{x}$. Similarly, the increase of $\mathrm{OH}$ exposure affects the major oxidation mechanism from functionalization to fragmentation, leading to the up and down trend of SOA yields. The higher seed particle loading provides more surface area for condensable products and then increases the SOA yields. The particle acidity favors the uptake process for gas-phase products and promote the SOA formation via further reactions in the condense phase. Trace components e.g. transition metals and minerals can be involved in the SOA formation and aging by catalysis or affecting the uptake of oxidants and their products. Chambers and PAM reactors are usually used to explore SOA formation potential of different sources. SOA formation potential from vehicles will be
\end{abstract}

*E-mail: songguo@pku.edu.cn

Received February 16, 2020; published May 25, 2020.

Project supported by the National Key R\&D Program of China (No. 2016YFC0202000), the National Natural Science Foundation of China (Nos. 51636003, 41977179, 21677002, 91844301) and the Open Research Fund of State Key Laboratory of Multi-phase Complex Systems (No. MPCS-2019-D-09).

项目受国家重点研发计划(No. 2016YFC0202000)、国家自然科学基金(Nos. 51636003, 41977179, 21677002, 91844301)和多相复杂系统国家重点实验室 开放课题(No. MPCS-2019-D-09)资助. 
influenced by engine types, engine loading and composition of fuel. The highest SOA enhancement ratio (SOA/POA) from gasoline engines is about $4 \sim 14$, when the equivalent photochemical days are $2 \sim 3 \mathrm{~d}$. The SOA production mass from gasoline vehicles is from about $10 \sim 40$ to $400 \sim 500 \mathrm{mg} / \mathrm{kg}$ fuel. The SOA formation potential is about $400 \sim 500 \mathrm{mg} / \mathrm{kg}$ fuel. The largest SOA enhancement ratio for biomass burning is $1.4 \sim 7.6$, which occurs at $3 \sim 4$ photochemical days. The SOA enhancement ratio from ambient air differs from region to region. However, the highest ratios all occur at the photochemical age of about $2 \sim 4 \mathrm{~d}$. We summarize the SOA characteristics evolution with aging. Oxidation state of particles will increase with $\mathrm{OH}$ exposure. Changes of $\mathrm{H} / \mathrm{C}$ and $\mathrm{O} / \mathrm{C}$ with increasing $\mathrm{OH}$ exposure can be plotted in the Van Krevelen diagrams. The slopes of fitted curve range from -1 to 0 , indicating OA evolution chemistry involving addition of carboxylic acids or addition of alcohols/peroxides. In addition, the volatility and hygroscopicity of oxidized OA will be higher than primary organic aerosols. In the future, more studies should be focused on developing new technologies to measuring the oxidized intermediate products at a molecular level. Also the researches on the mechanism of SOA formation from complex precursors are also crucial to understand the SOA formation at real atmosphere.

Keywords secondary organic aerosols; yield; formation potential; chambers; oxidation flow tube

\section{1 引言}

大气气溶胶对气候变化、区域污染及人体健康具有 重要的影响 ${ }^{[1]}$, 其中有机气溶胶 $(\mathrm{OA})$ 在气溶胶中的占比 可以达到 $20 \% \sim 90 \%{ }^{[2]}$, 是气溶胶中重要的组成部分. $\mathrm{OA}$ 来源复杂, 主要分为一次来源 $(\mathrm{POA})$ 和二次来源 $(\mathrm{SOA})^{[3]}$. 在我国一些城市的重污染期间, SOA 占 OA 的 比例约为 $44 \% \sim 71 \%{ }^{[4 \sim 7]}$. 但是, 目前对于 SOA 的估算 存在很大的低估. 有研究表明, 观测得到的 SOA 与模型 模拟的 SOA 相差几倍甚至几十倍 ${ }^{[8]}$. 因此, 对 SOA 估算 的不确定性是导致无法准确评估气溶胶对气候变化影 响的重要原因.

$\mathrm{SOA}$ 前体物的来源复杂, 生物源排放的各类挥发 性有机物(VOCs) 是 SOA 的重要前体物. 有研究表明, 从全球尺度来看生物源 VOCs 的氧化对 SOA 的贡献, 最 低估算值为 $20 \times 10^{12} \mathrm{~g} \cdot y e a r^{-1[9]}$, 最高估算值为 $380 \times$

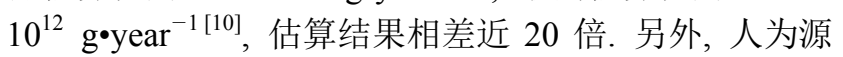
排放例如生物质燃烧、化石燃料燃烧及餐饮源排放等, 不仅会排放大量的 POA, 排放的气态有机物 (包括 VOCs、半/中等挥发性有机物 S/I VOCs) 也是 SOA 的重 要前体物 ${ }^{[5,11]}$.

$\mathrm{SOA}$ 的生成途径复杂, 总体来说主要分为气相反 应、非均相反应和液相反应三类途径, 三类反应途径相 互关联, 三者的关系如图 1 所示. 在气相中, 气态前体 物在 $\mathrm{OH}$ 自由基、 $\mathrm{O}_{3} 、 \mathrm{NO}_{3}$ 自由基和 $\mathrm{Cl}$ 等卤素原子等 的氧化作用下, 逐渐从高挥发性的物质演变为低挥发性 的物质, 进而通过气粒平衡分配到颗粒相, 即形成 $\mathrm{SOA}^{[12]}$. 气态前体物、气相反应的产物和氧化剂通过非 反应摄取和反应摄取进入颗粒相, 在颗粒物相中发生转 化, 从而保留在颗粒相形成 SOA 的过程, 称为非均相反 应. 其中在颗粒相中的转化途径, 又可以分为氧化途径 和非氧化途径. 氧化途径中, 一方面, 气相中的氧化剂 可以通过非均相摄取参与到颗粒相中物质的氧化 ${ }^{[12,13]}$, 另一方面, 颗粒相中的光敏剂或者金属离子, 可以作为 催化剂在光的作用下引发自由基链而产生氧化剂进而 参与颗粒相中的氧化反应 ${ }^{[14,15]}$. 非氧化途径中, 当颗粒 相在酸性条件或者存在 $\mathrm{NH}_{4}^{+}$的条件下, 气态前体物例
如异戊二烯的产物环氧二醇 (Isoprene Epoxydiol, IEPOX) 以及乙二醛等可以被摄取到颗粒相中发生非氧 化反应生成 $\mathrm{SOA}^{[16,17]}$; 此外, 分子间的酯化或者聚合反 应，也会产生更低挥发性的大分子量物质，从而保留在 颗粒相中 ${ }^{[18]}$; 另一方面, 已经在颗粒相中的 SOA, 经过 光照会逐渐分解, 重新进入到气相中 ${ }^{[19]}$.

目前, 对于 SOA 生成机制的研究主要通过外场观 测、实验室模拟和模式模拟等多种手段 ${ }^{[20,21]}$. 虽然基于 外场观测的方法是最直接的研究 SOA 生成及老化的方 法, 但是实际大气中条件复杂、受各种条件影响较多, 因此对研究 SOA 生成机制具有很大的困难; 另外目前 模型中估算 SOA 的参数, 均来自于实验室中的模拟. 因 此, 利用实验室模拟的手段对 SOA 生成机制的研究仍 然是目前较为常用的方法. 本综述将通过总结实验室模 拟方法研究 SOA 的生成机制及老化的进展, 探讨影响 SOA 产率的因素、不同源排放生成 SOA 的潜势以及 $\mathrm{SOA}$ 老化过程中性质的演变.

\section{2 基于实验室模拟的研究方法}

实验室模拟的方法，可以在相对稳定可控的条件 下，通过改变不同的反应变量从而探讨 SOA 的产率和 生成机制. 实验室中, 常用的两种反应器为烟雾箱和氧 化流动管 ${ }^{[22,23]}$. 两种反应器基本原理是: 在反应器中通 入 $\mathrm{SOA}$ 的气态前体物和氧化剂, 基于实验目的设计不 同的氧化剂浓度、 $\mathrm{NO}_{x}$ 浓度等, 探究在光反应条件或者 暗反应条件下, SOA 的生成潜势、反应机制和老化特

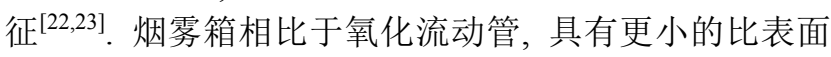
积(内表面积与体积的比), 可移动性差, 前体物的停留 时间及实验操作时间较长. 但是在氧化流动管中, 氧化 剂浓度更高, 虽然具有较短的停留时间, 也可以产生更 高的 $\mathrm{OH}$ 暴露量. 烟雾箱最高可以模拟数小时的光氧化 龄, 氧化流动管最高可以模拟数天的光氧化龄 ${ }^{[24]}$. 两种 反应器都可以较好地模拟 SOA 生成, 使用流动管可以 获得 SOA 的生成潜势, 即 SOA 的最大生成量, 但是更 高的氧化暴露可能会使 SOA 的生成机制与实际大气有 所不同. 


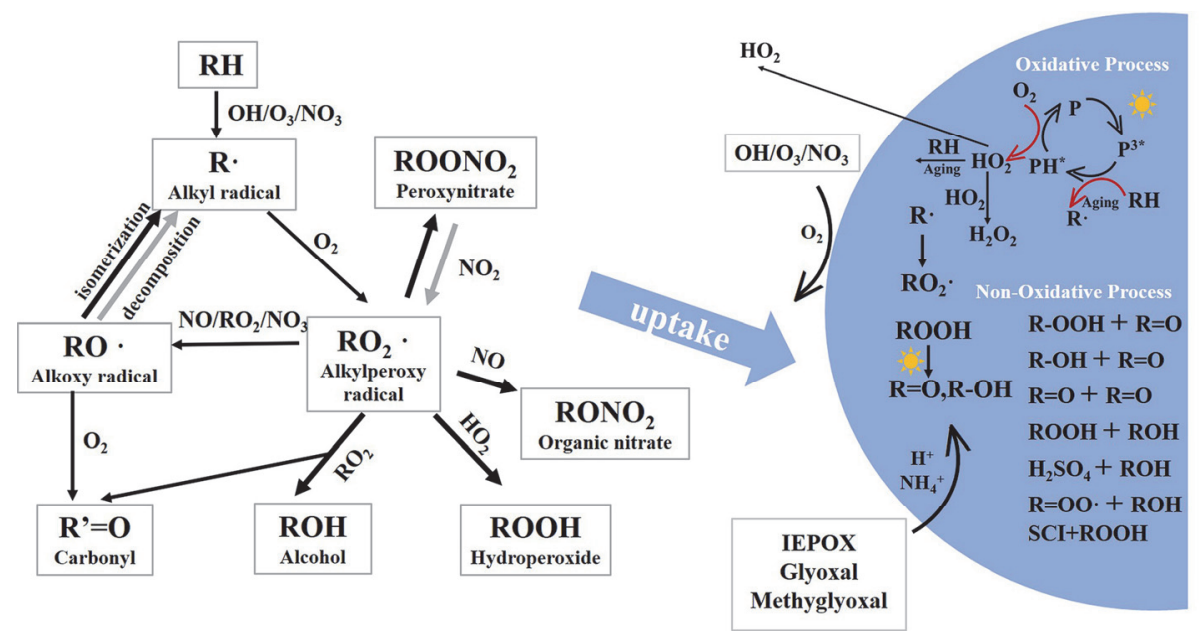

图 1 二次有机气溶胶的生成及老化途径

Figure 1 Process of secondary organic aerosol formation and aging

环境烟雾箱被认为是研究排放与空气质量关系最 为直接的方法之一. 在烟雾箱设计的过程中, 需要考虑 众多因素. 首先, 需要考虑光照的影响, 光源有自然光 源和人为光源两种. 其次, 材质的刚硬程度会影响烟雾 箱的造价、压力以及清洗难易程度, 材质的壁面效应对 反应器中反应的影响也是十分重要的. 研究表明, 一些 烟雾箱例如 SAPHIR 在光照的条件下, 会产生 HONO, 进而光解产生 $\mathrm{OH}$ 自由基, 从而成为重要的氧化剂来 源 ${ }^{[25]}$. 最后, 烟雾箱的体积表面积的比值也会通过影响 壁面损失从而影响反应的进行. 针对不同的科学问题, 众多研究组设计出了形态各异的烟雾箱, 表 1 列举了世 界上部分烟雾箱及其特征.

虽然烟雾箱能够较好地模拟 SOA 的生成及演变规 律, 但是由于烟雾箱中持续的壁损失, 使得反应物在烟 雾箱中的停留时间受到限制, 因此, 无法模拟更长时间 尺度的化学反应.

Kang 等 ${ }^{[23]}$ 在 2007 年首先提出了气溶胶生成潜势反 应器(Potential Aerosol Mass, 简称 PAM)的概念, 可以模 拟前体物生成 SOA 的过程, 进而模拟 SOA 的潜在最大 生成量, 也称为气溶胶生成潜势. 该反应器是氧化流动 管的一种, 其基本原理是通过在氧化器中提供高浓度的 氧化剂, 从而可以在较短的时间模拟较长光氧化龄条件 下的光化学反应. $\mathrm{OH}$ 暴露量 $(\mathrm{OH}$ Exposure) 是氧化流动 管的重要参数之一, 其计算方法如公式(1)所示 ${ }^{[26]}$, 此方 法基于 $\mathrm{SO}_{2}$ 氧化衰减的测定计算对应的 $\mathrm{OH}$ 暴露量, 其 中 $k_{\mathrm{OH}-\mathrm{SO} 2} \mathrm{OH}$ 与 $\mathrm{SO}_{2}$ 的反应速率常数 $9.0 \times 10^{-13}$ $\mathrm{cm}^{3} \cdot$ molecule ${ }^{-1} \cdot \mathrm{s}^{-1}, \mathrm{SO}_{2, \mathrm{i}}$ 为 $\mathrm{SO}_{2}$ 在反应器中初始的浓度, $\mathrm{SO}_{2, \mathrm{f}}$ 为 $\mathrm{SO}_{2}$ 在反应器中被 $\mathrm{OH}$ 自由基氧化后的浓度.

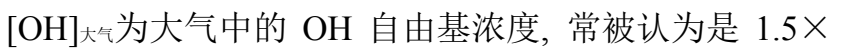
$10^{6}$ molecules $\cdot \mathrm{cm}^{-3}$, 进而可以通过公式(2)计算等效光 氧化龄(Equivalent Photochemical Age, EPA, 天).

$$
\mathrm{OH} \text { exposure }=\frac{-1}{k_{\mathrm{OH}-\mathrm{SO}_{2}}} \times \ln \left(\frac{\mathrm{SO}_{2, \mathrm{i}}}{\mathrm{SO}_{2, \mathrm{f}}}\right)
$$

Equivalent Photochemical Age $=\frac{\mathrm{OH} \text { exposure }}{24 \times 3600 \times[\mathrm{OH}] \text { 大气 }}$

图 2 总结了不同的研究中, 利用烟雾箱和 PAM 研 究单一前体物一 $\alpha$-蒎烯在不同的氧化剂暴露量下生成 SOA 的产率 ${ }^{[23,24,26-31]}$. 总结来看, 即使采用同样方法获 得的 $\alpha$-蒎烯 SOA 产率也存在差异, 这主要是由于在不 同的研究中, 实验条件如 $\mathrm{OH}$ 暴露量及种子颗粒物的存 在与否和具体浓度等因素都会存在差异. 在 Bruns 等 ${ }^{[24]}$ 的研究中发现, 在相似的氧化条件下, 烟雾箱和 PAM 获得的 $\alpha$-菠烯和生物质燃烧排放获得的 SOA 产率及性 质是相当的, 其差异小于实验自身的不确定性. 因此, 烟雾箱以及 PAM 都广泛被应用到对 SOA 的研究中.

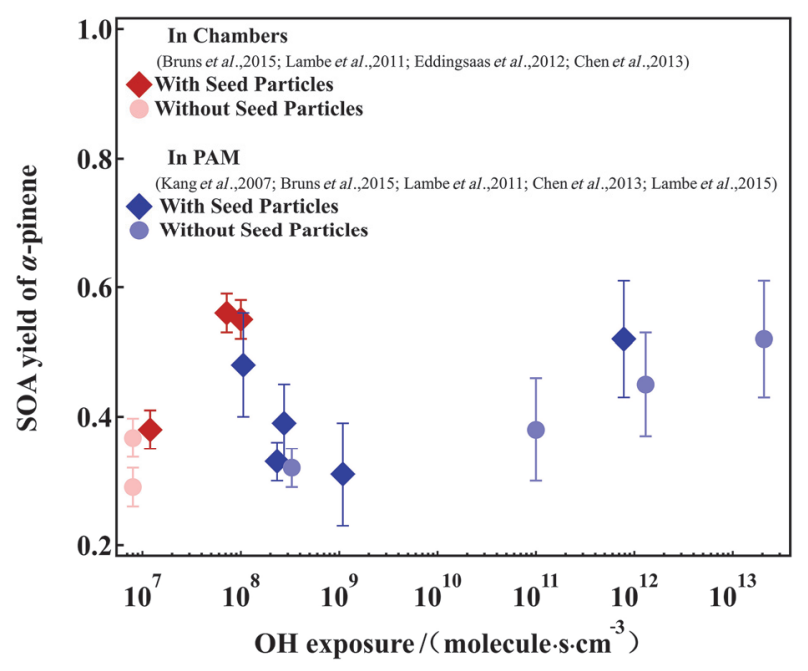

图 $2 \alpha$-蒎烯在不同反应器及 $\mathrm{OH}$ 暴露量下的 SOA 产率 $[23,24,26 \sim 31]$ Figure 2 SOA yield of $\alpha$-pinene as a function of $\mathrm{OH}$ exposure during different studies ${ }^{[23,24,26 \sim 31]}$ 
表 1 部分烟雾箱特征及主要研究应用

Table 1 The characteristics and major research of some chambers

\begin{tabular}{|c|c|c|c|c|}
\hline 名称 & 研究机构 & 体积 $/ \mathrm{m}^{3}$ & 材质 & 相关研究 \\
\hline $\begin{array}{l}\text { CLOUD (Cosmics Leaving } \\
\text { Outdoor Droplets) }\end{array}$ & University of Helsinki & 26.1 & Stainless Steel & $\begin{array}{l}\text { The formation mechanisms of aerosols }, \mathrm{CCN} \text { and ice } \\
\text { nuclei }^{[32,33]} \text {. }\end{array}$ \\
\hline CMU & Carnegie Mellon University & 12 & Teflon & Formation and aging of $\mathrm{SOA}^{[34,35]}$. \\
\hline $\begin{array}{l}\text { AIDA } \\
\text { (Aerosol Interaction and Dynam- } \\
\text { ics in the Atmosphere) }\end{array}$ & $\begin{array}{l}\text { Karlsruhe Institute of Tech- } \\
\text { nology }\end{array}$ & 84.5 & Aluminium & $\begin{array}{l}\text { Investigating the impact of aerosol on climate, weather } \\
\text { and the environment. }{ }^{[36,37]} \text {. }\end{array}$ \\
\hline $\begin{array}{l}\text { SAPHIR (Simulation of Atmos- } \\
\text { pheric Photochemistry } \\
\text { in a large Reaction chamber) }\end{array}$ & Juelich Forschungszentrum & 270 & Teflon & $\begin{array}{l}\text { Research about the atmospheric degradation of bio- } \\
\text { genic and anthropogenic trace gases and the build-up } \\
\text { of secondary particles and pollu- tants }{ }^{[25,38]} \text {. }\end{array}$ \\
\hline $\begin{array}{l}\text { CTEC (Caltech Teflon } \\
\text { Environmental Chambers) }\end{array}$ & Caltech & 24 & Teflon & $\begin{array}{l}\text { To investigate the chemical reaction kinemics of SOA } \\
\text { formation }^{[18,39]} \text {. }\end{array}$ \\
\hline $\begin{array}{l}\text { PACS-C3 } \\
\text { PSI Atmospheric Chemistry } \\
\text { Simulation Chambers }\end{array}$ & Paul Scherrer Institute & 27 & Teflon & $\begin{array}{l}\text { To investigate the oxidation products of different } \\
\text { precursors and then the SOA formation }{ }^{[30,40]} \text {. }\end{array}$ \\
\hline $\begin{array}{l}\text { EUPHORE European PHOtoRE- } \\
\text { actor }\end{array}$ & $\begin{array}{l}\text { Centro de Estudios Ambien- } \\
\text { tales del Mediterraneo }\end{array}$ & 200 & Teflon & To research atmospheric chemical process ${ }^{[41]}$. \\
\hline $\begin{array}{l}\text { Quasi-Atmospheric aerosol } \\
\text { evolution study (QUALITY) }\end{array}$ & Peking University & 1 & Teflon & $\begin{array}{l}\text { To investigate the formation of SOA and aging of } \\
\text { black carbon }^{[42,43]} \text {. }\end{array}$ \\
\hline LEAK-LACIS & $\begin{array}{l}\text { Leibniz Institute for Tropo- } \\
\text { spheric Research }\end{array}$ & 19 & Teflon & $\begin{array}{l}\text { Experimental and theoretical investigation of } \\
\text { gas-phase process, SOA formation process and process } \\
\text { of biomass burning exhausts as well as aerosol and } \\
\text { cloud microphysical process }{ }^{[4]]} \text {. }\end{array}$ \\
\hline
\end{tabular}

\section{3 实验室模拟二次有机气溶胶生成产率的影响 因素}

研究 SOA 生成最重要的一个参数就是产率, 同样 也是后续 SOA 估算模型中最重要的参数之一. SOA 产 率是指生成的 $\mathrm{SOA}$ 的质量与所消耗前体物质量的比值 $(\triangle \mathrm{MOA} / \triangle \mathrm{MHC})$, 生成 SOA 的前体物包括挥发性有机 气态物质以及半中等挥发性的物质等. 目前利用烟雾箱 模拟是获得 SOA 产率最直接和最有效的方法. 但是使 用烟雾箱等实验室的模拟手段, 对于产率的研究受到多 种因素的影响, 本部分总结了影响 SOA 产率的主要因 素, 可以分为两类: 一类是影响 SOA 生成的反应条件, 主要包括氧化剂水平、无机气态前体物 $\left(\mathrm{NO}_{x} 、 \mathrm{SO}_{2} 、 \mathrm{NH}_{3}\right)$ 、 种子颗粒物浓度及化学组成和相对湿度等: 另一类是由 于模拟方法中反应器的使用导致的壁损失.

氧化剂的暴露量是影响 SOA 产率的重要因素. Lambe 等 ${ }^{[30]}$ 发现随着 $\mathrm{OH}$ 暴露量的增加, $\alpha$-蒎烯的产率 先增加后降低, 最高产率出现在 $\mathrm{OH}$ 暴露量 $6 \times 10^{11}$ molecule $\cdot \mathrm{s}^{\circ} \mathrm{cm}{ }^{-3}$ 的条件下, 约为 0.35 . 一方面, 较高的 氧化剂条件促进了前体物的多代氧化, 提高了低挥发性 产物的产率, 进一步提高了 SOA 的产率 ${ }^{[45]}$. 另一方面, Jimenez 等 ${ }^{[3]}$ 的研究提出, 颗粒物的生成途径随着 $\mathrm{OH}$ 暴 露的增加会发生改变, 在暴露量增加到一定程度后, 已 生成的颗粒态 SOA 会发生分解, 从而使产率降低. 主要 原因是随着 $\mathrm{OH}$ 暴露量的增加, 主导反应途径逐渐由官 能团化, 转变为分子裂解, 从而产生了更多具有较高挥
发性的小分子产物，降低了 SOA 的产率 ${ }^{[46]}$.

主要来自于人为源排放的无机气态前体物, 包括 $\mathrm{NO}_{x} 、 \mathrm{SO}_{2}$ 和 $\mathrm{NH}_{3}$ 等, 也是影响 $\mathrm{SOA}$ 产率的重要因素. 一 方面, $\mathrm{NO}_{x}$ 的浓度水平可以直接影响有机物在气态中的 光化学氧化机制 ${ }^{[47,48]}$; 另一方面, 由 $\mathrm{NO}_{x} 、 \mathrm{SO}_{2}$ 和 $\mathrm{NH}_{3}$ 氧化反应生成的酸和盐会改变种子颗粒物的化学性质, 从而间接影响 SOA 的生成, 此部分将在总结种子颗粒 物的影响部分进行探讨 ${ }^{[49-51]}$.

$\mathrm{NO}_{x}$ 主要通过改变光化学反应速率和反应途径从 而影响 $\mathrm{SOA}$ 产率. 研究发现随着 $\mathrm{NO}_{x}$ 水平的提高, $\mathrm{SOA}$ 产率先增加后降低, 常用 $\mathrm{VOCs} / \mathrm{NO}_{x}$ 的比值来表征 $\mathrm{NO}_{x}$ 的相对水平. $\mathrm{NO}_{x}$ 的存在与否, 会改变 $\mathrm{RO}_{2}$ 自由基的归 趋, 在 $\mathrm{NO}_{x}$ 存在的条件下, 会促进自由基链的传递, 在 一定程度上促进前体物发生氧化反应. 在低 $\mathrm{NO}_{x}$ 条件 下, $\mathrm{RO}_{2}$ 主要与 $\mathrm{HO}_{2}$ 反应生成更低挥发性的物质 ${ }^{[47]}$, 促 进 $\mathrm{SOA}$ 的生成; 然而在高 $\mathrm{NO}_{x}$ 条件下, $\mathrm{RO}_{2}$ 与 $\mathrm{NO}_{x}$ 反应 产生 RO 自由基或者见光易分解的硝酸酯类物质，抑制 了 SOA 的生成 ${ }^{[49,52]}$. 但是这种影响, 在不同的前体物反 应体系下, 表现也会有所差异.

有大量研究证明, 生物源排放的前体物的 SOA 产 率会显著受到 $\mathrm{NO}_{x}$ 的影响. Kroll 等 ${ }^{[12]}$ 发现, 异戊二烯在 较低 $\mathrm{NO}_{x}$ 水平下, 随着 $\mathrm{NO}_{x}$ 的浓度先增加到一个稳定的 值, 稳定的值出现的 $\mathrm{VOCs} / \mathrm{NO}_{x}$ 的比值约为 $2.25: 1 \sim$ $0.75: 1$, 然后随着 $\mathrm{NO}_{x}$ 水平的继续增加而降低. Dommen 等 ${ }^{[33]}$ 则发现, $\mathrm{VOCs} / \mathrm{NO}_{x}$ 范围在 $25: 1 \sim 10.5: 1$ 时, 异戊二烯具有更低的 SOA 产率, 但是范围在 $10.5: 1$ 
$7: 1$ 时, 异戊二烯具有更高的 SOA 产率. Chan 等 ${ }^{[54]}$ 发 现, $\mathrm{VOCs} / \mathrm{NO}_{x}$ 比值在 $0.1: 1$ 到 $1: 1$ 时, $\mathrm{SOA}$ 产率逐渐 增加, 该比值继续增长后, $\mathrm{SOA}$ 的产率则开始降低. 在 较低的 $\mathrm{VOCs} / \mathrm{NO}_{x}$ 条件下, Chan 等 ${ }^{[54]}$ 获得的 $\mathrm{SOA}$ 产率 略高于 Kroll 等 ${ }^{[12]}$ 的结果, 主要由于 Chan 等 ${ }^{[54]}$ 使用的 $\mathrm{NO}_{2} / \mathrm{NO}$ 的比例与实际大气更为接近, 反应倾向于发生 多代反应, 从而促进了更低挥发性物质的产生. 单萜烯 氧化过程中也会受到 $\mathrm{NO}_{x}$ 水平的影响. Presto 等 ${ }^{[48]}$ 发现 $\mathrm{O}_{3}$ 氧化 $\alpha$-蒎烯在高 $\mathrm{NO}_{x}$ 条件下具有更低的 $\mathrm{SOA}$ 产生量. 但是, 当前体物含有两个双键时, 情况会更加复杂. 例 如, 柠檬烯在被 $\mathrm{O}_{3}$ 氧化的过程中, 低 $\mathrm{NO}_{x}$ 条件下, 非均 相的反应途径会促进 SOA 的生成, 高 $\mathrm{NO}_{x}$ 条件下, 气相 氧化途径成为主导 SOA 生成的途径 ${ }^{[55]}$.

人为源前体物 $\mathrm{SOA}$ 的生成产率也会受到 $\mathrm{NO}_{x}$ 浓度 水平的影响. 在低 $\mathrm{NO}_{x}$ 水平下, 对二甲苯和甲苯 $\mathrm{SOA}$ 的 产率分别约为 0.36 和 0.30 , 但是在高 $\mathrm{NO}_{x}$ 条件下, 二者 的产率约为 $0.10^{[52]}$. Zhao 等 ${ }^{[56]}$ 使用烟雾箱探究汽油车源 排放前体物随着 $\mathrm{NO}_{x}$ 水平变化的 $\mathrm{SOA}$ 的产率. $\mathrm{NMOG}$ (非甲烷有机气体)与 $\mathrm{NO}_{x}$ 的比值从 4 增长到 16 的过程 中, SOA 的产率逐渐增加并维持稳定. 总体来说, 无论 是生物源还是人为源前体物, 更高的 $\mathrm{NO}_{x}$ 条件均会使 $\mathrm{SOA}$ 产率降低, 这主要是由于 $\mathrm{RO}_{2}$ 与 $\mathrm{NO}_{x}$ 反应产生 $\mathrm{RO}$ 自由基或者见光易分解的硝酸酯类物质, 抑制了 SOA 的生成.

环境相对湿度的改变, 会显著影响气溶胶中的含水 量, 此平衡可以由 Köhler 方程描述 ${ }^{[56]}$, 气溶胶中的含水 量会进一步影响颗粒物中 SOA 的生成. 一方面, 气溶胶 中的水可以影响气态物质, 例如反应前体物和氧化剂的 摄取 ${ }^{[57 ~ 61]}$. 颗粒物表面存在的水会通过氢键的作用提 高物质的摄取，例如 Chan 等 ${ }^{[62]}$ 发现 $\mathrm{HO}_{x}$ 自由基的摄取 速率随着环境相对湿度的提高而显著提高. 含水量也可 以通过改变颗粒物的相态而影响物质在颗粒相中的扩 散速度, 从而改变物质的摄取系数 ${ }^{[60,61]}$. Thomas 等 ${ }^{[61]}$ 发 现在更高的相对湿度下, 臭氧的摄取系数比较低的相对 湿度高出几个数量级. $\mathrm{Li}$ 等 ${ }^{[63]}$ 发现, 在更高的相对湿度 下, $\alpha$-蒎烯产生的 SOA 在约为 $60 \%$ 的相对湿度下, 扩散 系数 $>10^{-12} \mathrm{~cm}^{2} \cdot \mathrm{s}^{-1}$, 而在约为 $25 \%$ 的相对湿度下, 扩 散系数 $<10^{-14} \mathrm{~cm}^{2} \cdot \mathrm{s}^{-1}$. 因此在高相对湿度下, SOA 的 反应损失约为 $60 \%$, 显著高于低相对湿度的 $20 \%$, 从而 降低了 SOA 的产率. 然而, 也有研究发现, 较高的相对 湿度可以通过降低在颗粒相中扩散的时间尺度, 提高在 颗粒相中的反应速率, 或者通过提高气态物质的反应摄 取，进而提高 $\alpha$-蒎烯生成 SOA 的产率 ${ }^{[64]}$.

另一方面, 气溶胶中的水还可以作为溶剂提供反应 体系, 或者直接参与到反应中, 因此颗粒相中的含水量 会通过影响反应途径和反应速率来影响 SOA 的产 率 ${ }^{[63,65]}$. 颗粒相中已有的 SOA 组分会被进一步氧化而 降低 SOA 的产率. Kroll 等 ${ }^{[65]}$ 发现, 在具有氧化条件的
环境中, 颗粒相中的有机物由于非均相氧化过程的发 生, $\mathrm{O} / \mathrm{C}$ 会提高, 但是碳链的断裂使得产物转化为具有 更高挥发性的小分子，从而降低了颗粒相中的有机物含 量. 在等效光氧化龄约为一周时，约有 $3 \% \sim 13 \%$ 的碳分 配到气相中. 含水量会通过影响颗粒相中的反应途径, 从而改变 $\mathrm{SOA}$ 的产物. 例如, Zhao 等 ${ }^{[59]}$ 以 $\mathrm{C}_{8} \mathrm{H}_{10} \mathrm{O}_{4}$, $\mathrm{C}_{8} \mathrm{H}_{10} \mathrm{O}_{4}$ 组分为主导的有机气溶胶, 在不同的相对湿度 下 $(30 \%, 60 \%, 90 \%)$ 以臭氧作为氧化剂氧化. 结果发现, 臭氧氧化均会使其产生不同程度的损耗，在高湿度条件 下，损失速率更大. 主要原因是臭氧与颗粒相中的有机 物形成的 Criegee 中间态产物, 会继续发生与水分子的 反应、分子内异构化或者去碳形成羧酸的反应，而相对 湿度的提高会促进与水分子的反应通道，氧化机制发生 了显著的改变. $\mathrm{RO}_{2}$ 自由基与 $\mathrm{RO}_{2}$ 自由基的反应会产生 不稳定的含四个氧的产物, 而含水量的增加会提高产物 的分解比例从而影响 SOA 的产生 ${ }^{[66]}$. 综上所述, 环境 相对湿度会影响颗粒相的含水量或者颗粒物的相态, 可 以改变气态前体物或者氧化剂的摄取系数, 或者影响颗 粒相中物质的反应机制而影响 SOA 的生成.

种子颗粒物的存在也会影响 SOA 的产率. 一方面, 种子颗粒物可以通过改变反应中间态产物的凝结汇，进 而影响 SOA 产率: 另一方面, 种子颗粒物中的化学组分 可以通过影响颗粒物的性质而影响有机物在颗粒相中 的反应而影响 $\mathrm{SOA}$ 的生成(硫酸和硫酸盐、 $\mathrm{NH}_{4}^{+}$、金 属及矿物质). 基于分配理论, 种子颗粒物的质量浓度 以及组分均会影响反应产物的气粒分配 ${ }^{[67]}$, 因此在模 拟过程中，不仅要考虑种子颗粒物的浓度，颗粒物的性 质也是非常重要的影响因素. 首先, 高浓度的种子颗粒 物可以提供较大的凝结汇来提高 SOA 的产率. Ehn 等 ${ }^{[68]}$ 指出， $\alpha$-蒎烯氧化后产生的具有不同挥发性的中间态产 物, 在颗粒物的初始形成及后续的增长过程中发挥着不 同程度的作用. 极低挥发性的有机物对 $1 \sim 100 \mathrm{~nm}$ 的颗 粒物均有贡献，随着粒径的增加，较高挥发性的物质逐 渐参与到颗粒物的增长过程中. $\mathrm{SO}_{2}$ 在大气中被氧化生 成气态硫酸, 气态硫酸是大气中成核继而增长成新粒子 的重要组分 ${ }^{[69 ~ 71]}$, 新粒子的生成为气态前体物提供了 凝结表面, 促进气相中间态有机产物转化到颗粒相生成 $\mathrm{SOA}^{[68]}$.

由 $\mathrm{SO}_{2}$ 经过二次反应转化, 在颗粒相中生成的硫酸 和硫酸盐等可以通过影响颗粒物的酸性性质, 改变气态 前体物的摄取或者反应途径，进而影响 SOA 的产物和 产率. 首先, 硫酸和硫酸盐的存在会影响 SOA 的组成. 例如, Liggio 等 ${ }^{[17]}$ 发现, 在使用硫酸铵和硫酸作为种子 颗粒物的条件下，乙二醛被摄取并产生有机硫酸酯.也 有大量研究表明，环氧化合物可以被酸性种子颗粒物发 生反应摄取，生成开环产物，继而在水分子和 $\mathrm{SO}_{4}^{2-}$ 的 作用下反应生成有机硫酸酯 ${ }^{[72,73]}$. 例如异戊二烯在低 $\mathrm{NO}_{x}$ 条件下的重要中间产物 IEPOX, 在酸催化反应摄取 
后环氧基打开, 进而与 $\mathrm{H}_{2} \mathrm{O}$ 、硫酸、2-甲基丁四醇以及 羟基硫酸酯等发生反应生成有机硫酸酯, 成为凝结相 $\mathrm{SOA}^{[50]}$. 第二, 硫酸和硫酸盐的存在会显著提高 SOA 的产率. Kang 等 ${ }^{[23]}$ 发现, PAM 中通入 $\mathrm{SO}_{2}$ 会使反应体系 中产生硫酸气溶胶, 使 $\alpha$-蒎烯的 SOA 产率提高 1.4 倍. Lambe 等 ${ }^{[30]}$ 发现, 在体系中分别加入相同浓度的硫酸铵 种子颗粒物或者酸性硫酸种子颗粒物, 酸性条件可以促 进 SOA 的生成. 戊二烯产率在中性种子颗粒物的环境, 约为 0.032 , 在酸性种子颗粒物的环境下, 产率可以被 提高到约 0.14. 并且, 随着种子颗粒物的浓度的逐渐升 高, SOA 产率也会逐渐提高.

$\mathrm{NH}_{3}$ 可以生成铵盐, 在颗粒相中以 $\mathrm{NH}_{4}^{+}$的形式存 在, 是大气气溶胶中主要的组分之一 ${ }^{[74]}$, 也是影响 SOA 生成的重要组分. 一方面, $\mathrm{NH}_{4}^{+}$可以作为催化剂促进 羰基化合物在颗粒相中的转化 ${ }^{[75 ~ 78]}$, 另一方面, 它也可 以直接参与到反应中 ${ }^{[16,79,80]}$, 从而影响 SOA 的生成. Nozière 等 ${ }^{[78]}$ 发现, $\mathrm{NH}_{4}^{+}$可以通过两种途径充当催化剂 催化乙二醛的转化, 其一, $\mathrm{NH}_{4}^{+}$可以作为 Bronsted 酸 (释放质子)或者形成共价氮化合物; 其二, $\mathrm{NH}_{4}^{+}$与乙二 醛发生亲核反应, 得到亚胺中间体, 进而形成杂环或者 低聚化合物. 研究进一步表明, $\mathrm{NH}_{4}^{+}$催化作用的参与, 不仅可以促进乙二醛转化形成乙缩醛, 也可以有效地提 高其他羰基化合物发生醇醛缩合反应，例如乙醛 等 ${ }^{[76,77]}$. $\mathrm{NH}_{4}^{+}$也可以直接与乙二醛发生反应, 产生具

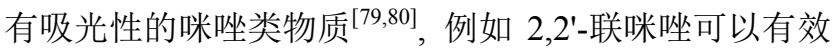
地吸收波长为 $280 \mathrm{~nm}$ 的紫外光 ${ }^{[80]}, 1 H$-咪唑-2-甲醛可以 有效地吸收波长为 $290 \mathrm{~nm}$ 的紫外光 ${ }^{[81]}$. 有研究进一步 发现, 此类吸光性物质可以作为光敏剂, 促进有机气态 前体物的摄取, 进而促进 SOA 的生成 ${ }^{[16]}$.

种子颗粒物中存在的金属离子也会通过直接或者 间接的作用, 影响有机物在颗粒相中的转化. 一方面, 金属离子可以直接作为催化剂, 促进有机物的转化. 作 为在颗粒相中存在丰度较高的金属元素铁, 离子 $\mathrm{Fe}^{3+}$ 会 与颗粒相中的有机物形成复合物, 在光化学的作用下, $\mathrm{Fe}^{3+}$ 转化为 $\mathrm{Fe}^{2+}$, 此过程也伴随着有机物配体的降 解 ${ }^{[82 \sim 85]}$. 有研究表明在上述过程中, $\mathrm{Fe}^{2+}$ 的量子产率受 到有机物配体、波长、配位数等因素的影响 ${ }^{[86,87]}$. 另一 方面, 金属离子可以影响氧化剂的生成和摄取, 从而为 有机物的氧化反应提供氧化剂, 进而影响 SOA 的生成. 在芬顿反应或者类芬顿反应中，低价金属例如二价铁、 三价铁等可以催化 $\mathrm{H}_{2} \mathrm{O}_{2}$ 分解生成 $\mathrm{OH}$ 自由基从而促进 有机物的氧化转化 ${ }^{[15,88]}$. Tofan-Lazar 等 ${ }^{[15]}$ 发现, 液相中 的邻二苯酚在 $\mathrm{Fe}^{3+}$ 存在的条件下, 可以有效地转化为 3,4,5-三羟基苯甲酸, 其产率随着邻二苯酚和 $\mathrm{Fe}^{3+}$ 的比 例的降低而升高. 也有研究表明, 金属离子可以通过催 化作用, 提高气相中 $\mathrm{HO}_{2}$ 自由基的摄取并转化为 $\mathrm{H}_{2} \mathrm{O}_{2}$, 从而为颗粒相中的反应提供氧化剂而影响 SOA 的反 应 ${ }^{[89,90]}$. 若将金属催化促进摄取机制考虑到模型中, 可 以更好地模拟 $\mathrm{HO}_{2}$ 的浓度水平 ${ }^{[91 ~ 93]}$.
种子颗粒物中矿物质例如半导体金属氧化物和无 机盐的存在, 也会影响 $\mathrm{SOA}$ 的生成 ${ }^{[94 ~ 99]}$. 在含有 $\mathrm{TiO}_{2}$ 和 $\mathrm{Fe}_{2} \mathrm{O}_{3}$ 等半导体金属氧化物的矿质气溶胶中，可以有 效地产生氧化剂, 为有机物在颗粒相中的氧化转化提供 基础 ${ }^{[95]}$. 也有研究发现, 矿物气溶胶在传输过程中会产 生大量的吸湿性组分例如硝酸钙, 因此会通过影响颗粒 物中的含水量而影响气态前体物的摄取和转化 ${ }^{[96 ~ 99]}$. Yu 等 ${ }^{[100]}$ 研究表明，甲苯、1,3,5-三甲基苯等汽油车排放的 有机气态前体物, 在沙尘作为种子颗粒物的体系中, 由 于羧酸盐的生成或者沙尘驱动的光催化氧化作用, SOA 的产率显著提高; 研究进一步表明, 在相对湿度更高的 体系, 由于沙尘中生成了具有吸湿性的硝酸盐, 提高了 颗粒物中的含水量, 因此 SOA 具有更高的产率. 其中颗 粒物的含水量对于 SOA 的产率的影响, 可参考前述章 节针对环境相对湿度影响 SOA 产率的总结.

相比于实际大气，反应器的壁损失是无可避免的， 也是造成产率不确定的另一个重要方面. 首先, 颗粒物 会直接损失到壁面上，会直接影响 SOA 的浓度从而降 低了对 SOA 产率的估算 ${ }^{[101]}$, 另一方面, 半挥发性组及 中等挥发性的有机气态物质(S/I VOVs)具有气粒分配性 质 ${ }^{[102]}$, 分配到壁面则降低了凝结到颗粒相中的比例, 从而降低了 SOA 的产率, 如图 3 所示.

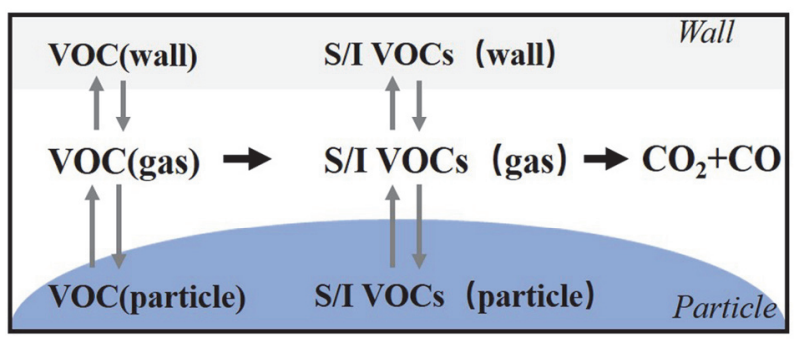

图 $3 \mathrm{~S} / \mathrm{I}$ VOCs 将分配在气相、颗粒相或者反应器壁面 ${ }^{[101]}$

Figure $3 \mathrm{~S} / \mathrm{I}$ VOCs will partition between gas and wall or particles ${ }^{[101]}$

大量研究基于颗粒物粒径进行颗粒物的损失校正, 来计算真实的 SOA 产率 ${ }^{[101]}$. 在最近的一项研究中, Zhang 等 ${ }^{[39]}$ 将半挥发性的物质在壁面上的损失考虑到模 型中后，发现如果不考虑半挥发性物质的损失，会大大 低估 SOA 的产率. 例如，甲苯产率在考虑壁损失时与未 考虑壁损失时的比值, 在高 $\mathrm{NO}_{x}$ 条件和低 $\mathrm{NO}_{x}$ 条件下, 分别是 $2.1 \sim 4.2$ 倍和 $2.1 \sim 3.6$ 倍 ${ }^{[39]}$. 因此, 若不考虑气 态中间产物损失的情况下, 获得的 SOA 产率在应用到 空气质量模型后, 会大大低估 SOA 的生成, 尤其是在人 为源为主导的地区. 当然, 颗粒物损失到壁面上的程度 会受到颗粒物化学组成的影响, 例如, 有机硝酸盐、碱 式硝酸盐以及酯类更容易损失到壁面上 ${ }^{[103]}$.

\section{4 不同源排放和实际大气中气态前体物生成 SOA 生成潜势}

气态前体物在氧化后可以生成 SOA 的最大量即为 
$\mathrm{SOA}$ 的生成潜势. SOA 的生成量随着在大气中光氧化龄 的增加, 先增加后降低, 获得的最高值即为 SOA 的生成 潜势. 研究中常使用 PAM 来对不同源气态前体物生成 SOA 的潜势进行分析, 如生物质燃烧、发动机排放、餐 饮源排放和其他源气体例如石油泄漏等, 并探究源排放 的改变对 SOA 生成的影响. 也有一些研究对复杂前体 物条件例如实际大气中的气态前体物生成二次颗粒物 的潜势开展研究, 这有助于对实际情况下生成颗粒物能 力的评估.

\section{1 机动车排放生成 SOA 潜势}

燃烧化石燃料会排放大量的有机气态前体物, 对 SOA 生成具有重要贡献. 化石燃料常常被用在发动机 中, 包括汽油发动机和柴油发动机. 研究表明在城市地 区, 机动车排放的 VOCs 是城市 SOA 的主要前体物 ${ }^{[104]}$; 而且生成的 SOA 可以通过与一次颗粒物相互作用, 影 响辐射和大气稳定度, 从而进一步促进颗粒物重污染形 成 ${ }^{[105]}$.

Tkacik 等 ${ }^{[106]}$ 在高速公路隧道中, 使用 PAM 提供光 氧化龄约为 $0.3 \sim 9.3 \mathrm{~d}$ 的氧化条件氧化道路中主要来自 机动车排放的实际大气. 在光氧化龄约为 $2 \sim 3 \mathrm{~d}$ 时, $\mathrm{SOA}$ 具有最高生成量, $\mathrm{SOA}$ 增长倍数约为 10 倍, $\mathrm{NO}_{x}$ 转 化为硝酸盐的效率约为 7\% 25\%. 随着 $\mathrm{OH}$ 暴露量的增 加, $\mathrm{OA}$ 逐渐从低氧化态转变为低挥发性高氧化的状态. 为了降低发动机的排放, 对油品的改变是正在探究的有 效方法之一. 例如，随着汽油中乙醇含量的增加 $(10 \%$ 、 $85 \%$ 及 $100 \%$ 一次排放的碳烃化合物浓度增加，芳香烃 的浓度及一次颗粒物浓度逐渐降低, SOA 的增长率逐渐 降低, 分别约为 13.4、1.5 和负增长 ${ }^{[107]}$. 发动机的类型 也会影响机动车排放对 SOA 的贡献, 近些年来, 各个国 家逐渐将发动机类型从进气道喷射式发动机(Port Fuel Injection, PFI)转变为汽油直接喷射式发动机(Gasoline Direct Injection, GDI), 针对发动机的转变, 美国和中国 的结果存在差异. 在美国, Zhao 等 ${ }^{[108]}$ 发现, 发动机的转 变, 并不会显著改变 SOA 的生成. 而排放标准的提升以 及老旧车辆的淘汰对降低 SOA 的生成具有更加显著的 改善. 但是在中国, Du 等 ${ }^{[43]}$ 发现, GDI 发动机的排放具 有更高的 SOA 生成量, 约为 PFI 的 2.7 倍. 这可能是由 于油品、循环工况和后处理装置的差异配合导致的. 汽 油车的负载也会影响 SOA 的生成, Zhang 等 ${ }^{[109]}$ 发现, 在 怠速条件下, 由于有机气态前体物的排放量更大, 相比 于匀速条件, SOA 的生成量更高, 怠速 $\mathrm{SOA}$ 产量约为 25 $\mathrm{mg} / \mathrm{kg}$ fuel, 匀速条件下约为 $10 \sim 15 \mathrm{mg} / \mathrm{kg}$ fuel, 并且随 着速度的增加, 产量逐渐降低. 因此, 汽油车排放生成 SOA 的产量会受到诸多因素影响, 导致不同研究中获 得的产量变化范围较大, 从约 $10 \sim 40$ 至 $40 \sim 500 \mathrm{mg} / \mathrm{kg}$ fuel ${ }^{[43,106,110 \sim 113]}$

Jathar 等 ${ }^{[114]}$ 对影响柴油发动机 SOA 的产率的因素 进行了探讨. 研究表明, 发动机的后处理装置虽然可以
降低一次有机气溶胶的排放, 但是具有后处理装置的情 况下, SOA 的增长率 $(80 \sim 800)$ 要远远高于无后处理装置 的情况 $(12 \sim 25)$. 急速条件下，一次颗粒物的排放和二 次有机气溶胶的生成均高于 $50 \%$ 的发动机负载. 欧盟国 家计划, 在 2020 年之前将使用生物柴油代替 $10 \%$ 的普 通柴油. Watne 等 ${ }^{[115]}$ 发现，压缩天然气及菜籽生物柴油 对 SOA 的生成并没有显著的影响, Jathar 等 ${ }^{[114]}$ 使用的生 物柴油和普通的柴油对比，也得出了类似的结论.

综上所述, 机动车排放生成 SOA 潜势受到多种因 素的影响，主要包括发动机类型、油品、运行条件等. 在 中国，未来普遍推广的GDI 发动机虽然可以提高汽油的 燃烧效率，但是由于油品和发动机配合的问题，却可能 具有更高的 SOA 生成潜势, 需要研究者针对中国的情 况进行进一步的探讨; 利用其它的替代油品例如乙醇会 有效降低 SOA 的生成潜势, 但是生物柴油的使用并没 有对 SOA 的生成产生显著的影响; 不同负载会影响尾 气生成二次颗粒物的化学组分, 负载越高, 有机物占比 越低.

\section{2 生物质燃烧排放生成 SOA 潜势}

在实际大气观测中, 燃烧的生物质类型以及燃烧过 程差异较大, 稀释状态受到实际大气温度湿度风场等的

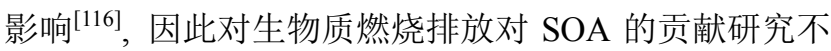
确定性较大. Ortega 等 ${ }^{[117]}$ 使用 PAM 提供稳定的氧化环 境，对美国包括黑衫木等 20 多种木质类型燃烧后 SOA 的生成潜势进行了对比. 其中，只有黄山松排放的颗粒 物浓度在氧化后，出现了下降的趋势. 其他木质类型燃 烧排放生成的 SOA 随着 $\mathrm{OH}$ 暴露均先增后减，最高 SOA 增长率出现在光氧化龄约为 $3 \sim 4 \mathrm{~d}$ 时候, 平均的 SOA 增长率 $1.42 \pm 0.36$. Fang 等 ${ }^{[118]}$ 在 $\mathrm{OH}$ 暴露量在 $(1.97 \sim 4.97) \times 10^{10}$ molecule $\cdot \mathrm{s}^{\bullet} \mathrm{cm}^{-3}$ 的条件下，探究露天 秙秆燃烧排放 SOA 的生成. 在该 $\mathrm{OH}$ 暴露量的范围内, SOA 的增长率逐渐增加, 从 2.4 倍逐渐增长到 7.6 倍, 产 率模型结果显示, 20 多种等主要气态有机前体物只能解 释约 5\% 27\%的 SOA 生成, 苯酚、苯二酚等具有较高 的贡献. 生物质含水量更高时，会增加润烧的时间，燃 烧温度和燃烧效率降低, 进而增加有机气态前体物的排 放 ${ }^{[119]}$ ，从而提高了 SOA 的产生量. 锅炉的燃烧效率也 会影响锅炉排放进而影响 SOA 的产率, 在特定的 $\mathrm{OH}$ 暴 露条件下 $\left(17.6 \times 10^{6}\right.$ molecule $\left.\cdot h \cdot \mathrm{cm}^{-3}\right)$, 改进后锅炉燃烧 排放具有更低的 SOA 产率, 从约 2.0 降为 $1.5^{[120]}$.

综上所述，生物质燃烧排放受到诸多因素的影响， 因此 SOA 的产生量也具有较大的差异. 其中, 生物质燃 烧类型、生物质含水量和燃烧条件会改变燃烧效率，进 而成为影响生物质燃烧排放的重要因素 ${ }^{[116,119,121,122] . ~}$

\section{3 其他源排放前体物生成 SOA 潜势}

除了上述针对不同的源气体进行的研究外，还有大 量研究针对其他源排放例如餐饮源，突发性事件造成的 
短暂性源排放例如石油泄漏等, 对 SOA 生成的贡献.

$\mathrm{Li}$ 等 ${ }^{[123]}$ 使用 PAM 对墨西哥湾泄露的石油排放, 对 SOA 生成贡献进行了探讨. 结果表明, 在实验开始阶 段, 较高挥发性的组分例如甲苯首先挥发出来, 而随着 实验的进行, 约持续 $3 \mathrm{~h}$ 后, 具有较低挥发性的组分 $\left(C^{*}=10^{5} \sim 10^{6} \mu \mathrm{g} \cdot \mathrm{m}^{-3}\right)$ 逐渐挥发出来, 并伴随着 SOA 的 大量生成及新粒子现象的发生. 虽然使用天然气发动机 之后, 排放的一次颗粒物浓度相比普通的汽油发动机显 著降低, 但是其却具有非常高的 SOA 生成潜势, SOA 的 增长比例最高达到 268 倍, 光氧化龄约为 $4.6 \sim 10.7 \mathrm{~d}$ 时, $\mathrm{SOA}$ 在气溶胶中的占比约为 $37 \% \sim 56 \%$, SOA 的生成潜 势约为 9 $20 \mathrm{mg} / \mathrm{kg}$ fuel ${ }^{[124]}$. 烹饪使用不同的食用油种 类的排放也会影响 $\mathrm{SOA}$ 的生成, 在 $\mathrm{OH}$ 暴露量从 $0 \sim 1.7$ $\times 10^{11}$ molecules $^{\bullet} \mathrm{s}^{\bullet} \mathrm{cm}^{-3}$ 变化的过程中, 不同食用油加 热挥发后的前体物产生的 SOA 随着 $\mathrm{OH}$ 暴露量的增加 而线性增长, $\mathrm{SOA}$ 的浓度从 $41 \sim 107 \mu \mathrm{g} \cdot \mathrm{m}^{-3}$ 增长到 $320 \sim 565 \mu \mathrm{g} \cdot \mathrm{m}^{-3}$, 具有非常高的 SOA 生成潜势. 其中 葵花油具有最高的 SOA 生成潜势, 挥发后的单体不饱 和脂肪和欧米伽- 6 脂肪酸对 SOA 具有重要贡献 ${ }^{[125]}$.

\section{4 实际大气中气态前体物生成 SOA 潜势}

实际大气中颗粒物及气体的时空分布具有较大差 异, 探究实际大气的后续氧化过程及性质的演变对于评 估气团的老化过程, 具有重要的意义. Saha 等 ${ }^{[126]}$ 对高速 公路旁边的夏季和冬季实际大气, 生成 SOA 的区别进 行研究. 在光氧化龄约为 $2 \sim 4 \mathrm{~d}$ 时, SOA 增长达到最大 值, 冬季生成 SOA 浓度 $\left(0.5 \sim 1 \mu \mathrm{g} \bullet \mathrm{m}^{-3}\right)$ 低于夏季 $(3 \sim 8$ $\left.\mu \mathrm{g} \cdot \mathrm{m}^{-3}\right)$. 虽然机动车的排放差异可以解释一部分 SOA 生成的季节间差异, 但是夏季更高的生物源排放可以更 好地解释上述差异. Liu 等 ${ }^{[127]}$ 使用等效光氧化龄约为 $0 \sim 14 \mathrm{~d}$ 的模拟氧化环境, 对比洛杉矶和北京实际大气 的 SOA 生成潜势. 两个城市的大气被氧化后, SOA 生成 量随着 $\mathrm{OH}$ 暴露量的增加均先增加后降低. 北京出现最 高 SOA 生成量对应的光氧化龄(约 $4 \mathrm{~d}$ )晚于洛杉矶(约 2 d), 并且北京实际大气在氧化后, SOA 的浓度整体偏高, 北京实际大气中更高的前体物浓度可能是造成此结果 的重要因素.

亚马逊地区, 以生物源前体物为主导, 全年分为干 湿两季(干燥季节相对湿度约为 $87 \%$, 湿润季节相对湿 度约为 $92 \%$ ). 干燥季节, SOA 生成的质量约为湿润季节 的 4 5 倍, 其中干燥季节来自于城市地区的生物质燃 烧等人为源的前体物是造成差异的重要原因. 光氧化龄 超过 1 4 d 之后, 随着 $\mathrm{OH}$ 暴露量的增加, $\mathrm{OA}$ 浓度逐渐 降低, 产物的裂解或者非均相反应的发生, 是导致 SOA 产率降低的重要原因 ${ }^{[128]}$. 白翎岛的气团, 由于风场风 向的改变, 可能来自中国和朝鲜半岛. Kang 等 ${ }^{[129]}$ 发现, 来自中国的长距离传输气团含有大量的 $\mathrm{SO}_{2}$, 经过 PAM 氧化后产生大量的硫酸盐颗粒物, 而来自朝鲜半岛的短 距离传输气团含有大量的 VOCs, 经过 PAM 氧化后, 产
生更多的 SOA.

\section{5 二次有机气溶胶老化过程中性质演变}

颗粒物通过气固分配、非均相反应、凝结相反应以 及碰并等一系列的物理化学反应方式后，其理化特征发 生改变, 此过程被称为老化. 在实际大气中, 颗粒有机 物会逐渐老化, 有机物的性质会随之改变. Jimenez 等 ${ }^{[3]}$ 指出, 颗粒物氧化性、挥发性以及吸湿性都会发生改变. 颗粒物性质的演变一方面会影响 SOA 的进一步反应和 气态中间产物的气粒分配, 另一方面, 颗粒物性质的演 变也是影响全球辐射强迫的重要因素. 因此使用烟雾箱 或者 PAM 来探究颗粒物随着 $\mathrm{OH}$ 暴露量增加的性质演 变, 进而探究如何将其在模型中准确表达成为了研究者 们关注的重点.

利用气溶胶质谱技术可以获得颗粒物中物质的分 子碎片以及元素信息, 其中质荷比为 43 和 44 的分子碎 片占比, $\mathrm{O} / \mathrm{C}$ 和 $\mathrm{H} / \mathrm{C}$, 常作为表征颗粒物的氧化态的重 要参数. 在氧化过程中, $\mathrm{O} / \mathrm{C}$ 逐渐增加, $\mathrm{H} / \mathrm{C}$ 逐渐降低, 氧化过程中, 二者关系拟合的曲线斜率可以表征有机气 溶胶氧化的机制 ${ }^{[130]}$.

图 4 总结了部分文献中获得的 $\mathrm{H} / \mathrm{C}$ 及 $\mathrm{O} / \mathrm{C}$ 随着 $\mathrm{OH}$ 暴露量的变化图. 在每个研究中选择了最高与最低的 $\mathrm{O} / \mathrm{C}$ 和 $\mathrm{H} / \mathrm{C}$ 进行绘图, 并依据 $\mathrm{OH}$ 暴露量的大小进行染 色 $^{[117,118,125 ~ 127,129,131]}$. 随着 $\mathrm{OH}$ 暴露量的增加, $\mathrm{O} / \mathrm{C}$ 逐渐 增加, $\mathrm{H} / \mathrm{C}$ 逐渐降低, 氧化程度逐渐升高, 绝对值的差异 主要来源于 $\mathrm{OH}$ 暴露量和前体物的差异. 斜率的变化范 围在 $-1 \sim 0$ 之间，主要的氧化机制为增加 $-\mathrm{OH},-\mathrm{OOH}$, 或者生成羧酸基团.

常用热解吸技术对颗粒物中物质的挥发性进行分 析. 颗粒物被固定温度加热一段时间后，剩余体积的大 小可以反应颗粒物组成物质的挥发性的高低. 剩余量越 大，说明颗粒物中的低挥发性组分占比越高 ${ }^{[132]}$. Saha 等 ${ }^{[126]}$ 发现，使用 $60{ }^{\circ} \mathrm{C}$ 和 $120{ }^{\circ} \mathrm{C}$ 对实际大气氧化产生 的 SOA 进行加热后, 随着光氧化龄的增加, 颗粒物的体 积剩余量先降低后增加, 在光氧化龄为 $2 \sim 3 \mathrm{~d}$ 时, 达到 最低值, 说明生成的物质挥发性先增加后降低. 颗粒物 的吸湿性参数 $\kappa$ 值, 是表征颗粒物的活化成云凝结核能 力的重要参数. Lambe 等 ${ }^{[31]}$ 发现, 间二甲苯和 $\alpha$-蒎烯氧 化后, 产生的 $50 \mathrm{~nm}$ 颗粒物随着 $\mathrm{OH}$ 暴露量的增加, 氧 化态逐渐增加, 稀释参数逐渐增大, 表明颗粒物形成云 凝结核的能力增强. 生物质燃烧排放, 不论一次排放还 是二次生成的有机物, 都含有大量的吸光的棕色碳. 相 比于一次颗粒物，二次生成的棕色碳具有更大的波长依 赖性，在近紫外光和短波段的可见光区域，具有更强的 吸光性, 从而影响了全球的辐射强迫 ${ }^{[133]}$.

\section{6 总结与展望}

$\mathrm{SOA}$ 对全球有机气溶胶具有重要的贡献. 利用实 


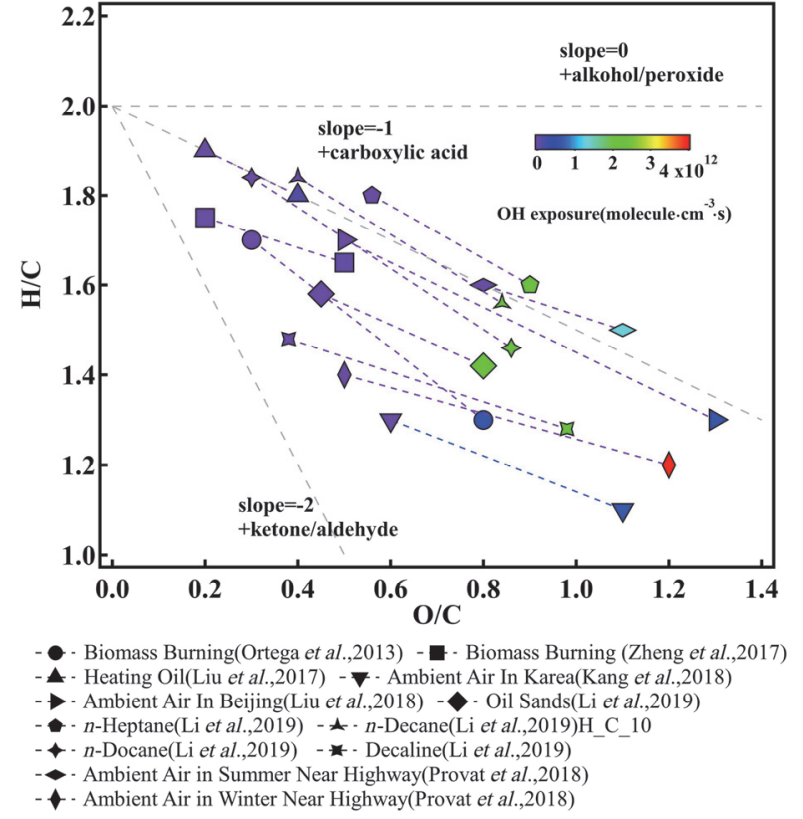

图 4 总结不同研究中 $\mathrm{O} / \mathrm{C}$ 及 $\mathrm{H} / \mathrm{C}$ 随 $\mathrm{OH}$ 暴露量的变 化 ${ }^{[117,118,125 \sim 127,129,131]}$

Figure 4 The summary of $\mathrm{O} / \mathrm{C}$ and $\mathrm{H} / \mathrm{C}$ changing with $\mathrm{OH}$ exposure in different studies ${ }^{[117,118,125 \sim 127,129,131]}$

验室的研究手段, 借助烟雾箱及气溶胶生成潜势反应器 可以在稳定和可控的条件下探究 SOA 的生成机制及老 化过程的性质演变.

在本篇综述中, 首先我们总结了影响 SOA 生成产 率的因素. 随着 $\mathrm{OH}$ 暴露量的增加, SOA 主导生成机制 由官能团化转变为分子裂解, 因此产率先增后降; 随着 $\mathrm{NO}_{x}$ 水平的提高, $\mathrm{SOA}$ 的产率先增加后降低, 在高 $\mathrm{NO}_{x}$ 条件下, 由于 $\mathrm{RO}_{2}$ 自由基倾向于与 $\mathrm{NO}$ 或者 $\mathrm{NO}_{2}$ 反应生 成硝酸酯类物质而降低了 SOA 的产率; 环境相对湿度 的改变会影响颗粒物中的含水量, 含水量的变化会通过 影响气态前体物和氧化剂的摄取、影响颗粒相有机物的 转化速率和转化途径等来影响 SOA 的生成和老化: 高 浓度的种子颗粒物为中间产物提供了更大的凝结汇, 提 高了 SOA 的产率. 酸性种子颗粒物会通过酸催化反应 途径促进气态前体物例如 IEPOX 等的摄取, 进而在颗 粒相中转化生成 SOA; 种子颗粒物中的变价金属或者 矿物质, 可以通过催化作用、影响氧化剂的摄取和产生 以及改变种子颗粒物的性质等方面来影响 $\mathrm{SOA}$ 的生成; 反应器的使用不可避免地带来了颗粒物和中间产物的 壁损失, 从而降低了 SOA 的生成. 其次, 我们总结了不 同源排放气体 SOA 的生成产率和潜势以及影响因素. 发动机类型、发动机运行条件和油品等会通过改变机动 车的排放而影响其对 SOA 的贡献, 这也是探讨如何从 源排放角度削减 SOA 生成的重要依据; 基于不同的研 究获得的生物质燃烧排放产生的 SOA 具有较大的差异, 燃烧条件的可控性差是导致 SOA 产率差异的重要因素; 其他源例如餐饮源排放、石油泄漏等也都是 $\mathrm{SOA}$ 生成
的重要来源; 实际大气的季节性和地域性差异是影响 $\mathrm{SOA}$ 生成的重要因素. 最后, SOA 的性质会随着颗粒物 老化的进行而改变, 随着 $\mathrm{OH}$ 暴露量的增加, $\mathrm{SOA}$ 的氧 化态逐渐增加, 氧化过程以增加羟基、过氧羟基及羧酸 基团为主，挥发性先增加后降低，吸湿能力逐渐增强， 会产生依赖于波长的吸光性物质.

综上所述, 本文针对使用烟雾箱及气溶胶生成潜势 反应器研究 SOA 的生成及老化做出以下展望: SOA 生 成过程中中间物种的测量技术开发、复杂体系下 SOA 生成机制的研究和 SOA 演化过程中特性的表征是未来 SOA 研究的重要方面.

(1)进一步完善 SOA 的生成机制: 开发新的测量技 术对 SOA 生成过程中的中间产物进行测量. 更加准确 认知对 SOA 产生贡献的前体物种类, 大量研究表明, VOCs 对 SOA 的贡献远不及 S/I VOCs 对于 SOA 的贡献. 但是目前, 对于 S/I VOCs 的源谱并不明确, 缺少对源谱 的定性定量分析. 提高对前体物在氧化过程中氧化机制 的认识，不仅包括单一前体物氧化的环境，还包括混合 条件的氧化环境. 例如单一前体物在氧化过程中的一代 反应产物, 以及二代反应可能进行的途径. 在生物源与 人为源共同作用的环境例如北京实际大气，如何评估 $\mathrm{SOA}$ 在此类环境中的生成及老化成为难点.

(2)复杂体系下 SOA 生成机制的研究需要加强. 基 础理论需要进一步基于实际大气情况进行完善. 前体物 在氧化过程中, 挥发性会逐渐发生变化, 但是如何将挥 发性的演变进行参数化, 进而应用到估算 SOA 的模型 中成为难点. 目前, 基于非均相反应的理论也不够明确, 比如中间产物在颗粒相中的反应摄取的热动力学参数 的计算等, 导致还无法合理地模拟非均相氧化的进行.

(3)对 SOA 演化过程中特征的表征, 需要进一步加 强. 针对 SOA 对于气候效应的评价, 具有较大的不确定 性, 很大部分的原因在于对 SOA 的性质没有准确的参 数化表征. 例如在前述总结中, 在不同的研究中 $\mathrm{O} / \mathrm{C}$ 等 在氧化后差异较大, 并且氧化途径也各不相同, 挥发性 的演变也存在较大差异. 因此探究及参数化 SOA 老化 后的性质, 对于改善模型具有重要的意义.

\section{作者简介}

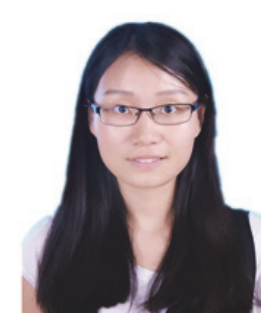

王辉, 博士研究生, 2017 年进入北京大学环境科学与工程 学院. 主要研究方向: 大气细颗粒物的来源及化学组成, 实验 室模拟研究二次有机气溶胶的生成机制. 


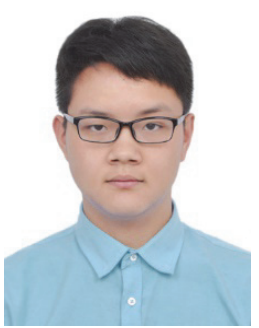

俞颖, 硕士研究生. 2019 年获得北京大学环境科学与工程 学院本科学位. 主要研究方向: 挥发性有机物来源与活性研 究、典型源的二次气溶胶生成潜势和特征研究.

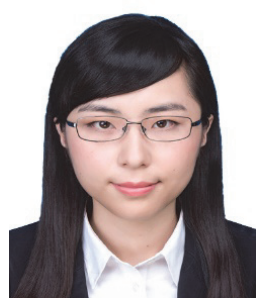

唐荣志, 博士研究生. 2016 年进入北京大学环境科学与工 程学院. 主要研究方向: 大气中的半挥发性有机物的测量及 对二次有机气溶胶的生成的贡献, 二次有机气溶胶的组成及 来源分析.

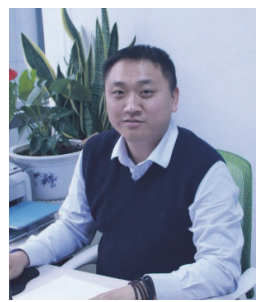

郭松, 博士, 北京大学环境科学与工程学院青年千人计 划研究员, 博士生导师. 2005 年和 2011 年分别获得北京大学 学士学位和博士学位, 2015 年获得北京大学职位. 主要研究方 向为大气气溶胶二次转化机制及其环境效应, 具体包括二次 有机气溶胶组成及生成机制、黑碳气溶胶老化机制及其环境 效应等。承担和参与多项国家自然科学基金委项目和国家重 点研发计划项目.

\section{References}

[1] Pope, C. A.; Dockery, D. W. J. Air Waste Manag. Assoc. 2006, 56, 709.

[2] Zhang, Q.; Jimenez, J. L.; Worsnop, D. R.; Canagaratna, M. Environ. Sci. Technol. 2007, 41, 3213.

[3] Jimenez, J. L.; Canagaratna, M. R.; Donahue, N. M.; Prevot, A. S. H.; Zhang, Q.; Kroll, J. H.; DeCarlo, P. F.; Allan, J. D.; Coe, H.; Ng, N. L.; Aiken, A. C.; Docherty, K. S.; Ulbrich, I. M.; Grieshop, A. P.; Robinson, A. L.; Duplissy, J.; Smith, J. D.; Wilson, K. R.; Lanz, V. A.; Hueglin, C.; Sun, Y. L.; Tian, J.; Laaksonen, A.; Raatikainen, T.; Rautiainen, J.; Vaattovaara, P.; Ehn, M.; Kulmala, M.; Tomlinson, J. M.; Collins, D. R.; Cubison, M. J.; Dunlea, E. J.; Huffman, J. A.; Onasch, T. B.; Alfarra, M. R.; Williams, P. I.; Bower, K.; Kondo, Y.; Schneider, J.; Drewnick, F.; Borrmann, S.; Weimer, S.; Demerjian, K.; Salcedo, D.; Cottrell, L.; Griffin, R.; Takami, A.; Miyoshi, T.; Hatakeyama, S.; Shimono, A.; Sun, J. Y.; Zhang, Y. M.; Dzepina, K.; Kimmel, J. R.; Sueper, D.; Jayne, J. T.; Herndon, S. C.; Trimborn, A. M.; Williams, L. R.; Wood, E. C.; Middlebrook, A. M.; Kolb, C. E.; Baltensperger, U.; Worsnop, D. R.; Stockholms, U. Science 2009, 326,1525 .

[4] Huang, R. J.; Zhang, Y.; Bozzetti, C.; Ho, K. F.; Cao, J. J.; Han, Y.;
Daellenbach, K. R.; Slowik, J. G.; Platt, S. M.; Canonaco, F.; Zotter, P.; Wolf, R.; Pieber, S. M.; Bruns, E. A.; Crippa, M.; Ciarelli, G.; Piazzalunga, A.; Schwikowski, M.; Abbaszade, G.; Schnelle-Kreis, J.; Zimmermann, R.; An, Z.; Szidat, S.; Baltensperger, U.; El Haddad, I.; Prevot, A. S. Nature 2014, 514, 218.

[5] Tang, R. Z.; Wu, Z. P.; Li, X.; Wang, Y. J.; Shang, D. J.; Xiao, Y.; Li, M. R.; Zeng, L. M.; Wu, Z. J.; Hallquist, M.; Hu, M.; Guo, S. Atmos. Chem. Phys. 2018, 18, 4055.

[6] Guo, S.; Hu, M.; Guo, Q.; Zhang, X.; Schauer, J. J.; Zhang, R. Atmos. Chem. Phys. 2013, 13, 8303 .

[7] Hu, M.; Guo, S.; Peng, J. F.; Wu, Z. J. Natl. Sci. Rev. 2015, 3, 257.

[8] Volkamer, R.; Jimenez, J. L.; Martini, F. S.; Dzepina, K.; Zhang, Q.; Salcedo, D.; Molina, L. T.; Worsnop, D. R.; Molina, M. J. Geophys. Res. Lett. 2006, 33,

[9] Kanakidou, M.; Seinfeld, J. H.; Pandis, S. N.; Barnes, I.; Dentener, F. J.; Facchini, M. C.; Van Dingenen, R.; Ervens, B.; Nenes, A.; Nielsen, C. J.; Swietlicki, E.; Putaud, J. P.; Balkanski, Y.; Fuzzi, S.; Horth, J.; Moortgat, G. K.; Winterhalter, R.; Myhre, C. E. L.; Tsigaridis, K.; Vignati, E.; Stephanou, E. G.; Wilson, J. Atmos. Chem. Phys. 2005, 5, 1053.

[10] Spracklen, D. V.; Jimenez, J. L.; Carslaw, K. S.; Worsnop, D. R.; Evans, M. J.; Mann, G. W.; Zhang, Q.; Canagaratna, M. R.; Allan, J.; Coe, H.; McFiggans, G.; Rap, A.; Forster, P. Atmos. Chem. Phys. 2011, 11, 12109.

[11] Guo, S.; Hu, M.; Guo, Q.; Zhang, X.; Zheng, M.; Zheng, J.; Chang, C. C.; Schauer, J. J.; Zhang, R. Environ. Sci. Technol. 2012, 46, 9846.

[12] Kroll, J. H.; Ng, N. L.; Murphy, S. M.; Flagan, R. C.; Seinfeld, J. H. Environ. Sci. Technol. 2006, 40, 1869.

[13] Zhou, S.; Joudan, S.; Forbes, M. W.; Zhou, Z.; Abbatt, J. P. D. Environ. Sci. Technol. Lett. 2019, 6, 243.

[14] Corral Arroyo, P.; Bartels-Rausch, T.; Alpert, P. A.; Dumas, S.; Perrier, S.; George, C.; Ammann, M. Environ. Sci. Technol. 2018, 52, 7680 .

[15] Tofan-Lazar, J.; Situm, A.; Al-Abadleh, H. A. J. Phys. Chem. A 2013, 117, 10368

[16] Rossignol, S.; Aregahegn, K. Z.; Tinel, L.; Fine, L.; Noziere, B.; George, C. Environ. Sci. Technol. 2014, 48, 3218.

[17] Liggio, J.; Li, S.-M.; McLaren, R. Environ. Sci. Technol. 2005, 39, 1532 .

[18] Kenseth, C. M.; Huang, Y.; Zhao, R.; Dalleska, N. F.; Hethcox, J. C.; Stoltz, B. M.; Seinfeld, J. H. Proc. Natl. Acad. Sci. U. S. A. 2018 115,8301 .

[19] Epstein, S. A.; Blair, S. L.; Nizkorodov, S. A. Environ. Sci. Technol. 2014, $48,11251$.

[20] Guo, S.; Hu, M.; Shang, D.; Guo, Q.; Hu, W. Acta Chim. Sinica 2014, 72, 145. (郭松, 胡敏, 尚冬杰, 郭庆丰, 胡伟伟, 化学学报, 2014, 72, 145.)

[21] Guo, S.; Hu, M.; Guo, Q.; Shang, D. Acta Chim. Sinica 2014, 72, 658. (郭松, 胡敏, 郭庆丰, 尚冬杰, 化学学报, 2014, 72, 658.)

[22] Kleindienst, T. E.; Jaoui, M.; Lewandowski, M.; Offenberg, J. H.; Lewis, C. W.; Bhave, P. V.; Edney, E. O. Atmos. Environ. 2007, 41, 8288.

[23] Kang, E.; Root, M. J.; Toohey, D. W.; Brune, W. H. Atmos. Chem. Phys. 2007, 7, 5727.

[24] Bruns, E. A.; El Haddad, I.; Keller, A.; Klein, F.; Kumar, N. K.; Pieber, S. M.; Corbin, J. C.; Slowik, J. G.; Brune, W. H.; Baltensperger, U.; Prévôt, A. S. H. Atmos. Meas. Tech. 2015, 8, 2315.

[25] Rohrer, F.; Bohn, B.; Brauers, T.; Brüning, D.; Johnen, F. J.; Wahner, A.; Kleffmann, J. Atmos. Chem. Phys. 2005, 5, 2189.

[26] Kang, E.; Toohey, D. W.; Brune, W. H. Atmos. Chem. Phys. 2011, $11,1837$.

[27] Lambe, A. T.; Ahern, A. T.; Williams, L. R.; Slowik, J. G.; Wong, J. P. S.; Abbatt, J. P. D.; Brune, W. H.; Ng, N. L.; Wright, J. P.; Croasdale, D. R.; Worsnop, D. R.; Davidovits, P.; Onasch, T. B. Atmos. Meas. Tech. 2011, 4, 445.

[28] Eddingsaas, N. C.; Loza, C. L.; Yee, L. D.; Chan, M.; Schilling, K. A.; Chhabra, P. S.; Seinfeld, J. H.; Wennberg, P. O. Atmos. Chem. Phys. 2012, 12, 7413.

[29] Chen, S.; Brune, W. H.; Lambe, A. T.; Davidovits, P.; Onasch, T. B. Atmos. Chem. Phys. 2013, 13, 5017.

[30] Lambe, A. T.; Chhabra, P. S.; Onasch, T. B.; Brune, W. H.; Hunter, J. F.; Kroll, J. H.; Cummings, M. J.; Brogan, J. F.; Parmar, Y.; Worsnop, D. R.; Kolb, C. E.; Davidovits, P. Atmos. Chem. Phys. 2015, 15, 3063 .

[31] Lambe, A. T.; Onasch, T. B.; Massoli, P.; Croasdale, D. R.; Wright, J. P.; Ahern, A. T.; Williams, L. R.; Worsnop, D. R.; Brune, W. H.; 
Davidovits, P. Atmos. Chem. Phys. 2011, 11, 8913.

[32] Kirkby, J.; Duplissy, J.; Sengupta, K.; Frege, C.; Gordon, H.; Williamson, C.; Heinritzi, M.; Simon, M.; Yan, C.; Almeida, J.; Trostl, J.; Nieminen, T.; Ortega, I. K.; Wagner, R.; Adamov, A.; Amorim, A.; Bernhammer, A.-K.; Bianchi, F.; Breitenlechner, M.; Brilke, S.; Chen, X.; Craven, J.; Dias, A.; Ehrhart, S.; Flagan, R. C.; Franchin, A.; Fuchs, C.; Guida, R.; Hakala, J.; Hoyle, C. R.; Jokinen, T.; Junninen, H.; Kangasluoma, J.; Kim, J.; Krapf, M.; Kurten, A.; Laaksonen, A.; Lehtipalo, K.; Makhmutov, V.; Mathot, S.; Molteni, U.; Onnela, A.; Perakyla, O.; Piel, F.; Petaja, T.; Praplan, A. P.; Pringle, K.; Rap, A.; Richards, N. A. D.; Riipinen, I.; Rissanen, M. P.; Rondo, L.; Sarnela, N.; Schobesberger, S.; Scott, C. E.; Seinfeld, J. H.; Sipila, M.; Steiner, G.; Stozhkov, Y.; Stratmann, F.; Tomé, A.; Virtanen, A.; Vogel, A. L.; Wagner, A. C.; Wagner, P. E.; Weingartner, E.; Wimmer, D.; Winkler, P. M.; Ye, P.; Zhang, X.; Hansel, A.; Dommen, J.; Donahue, N. M.; Worsnop, D. R.; Baltensperger, U.; Kulmala, M.; Carslaw, K. S.; Curtius, J.; Stockholms, U. Nature 2016, 533, 521 .

[33] Trostl, J.; Chuang, W. K.; Gordon, H.; Heinritzi, M.; Yan, C.; Molteni, U.; Ahlm, L.; Frege, C.; Bianchi, F.; Wagner, R.; Simon, M.; Lehtipalo, K.; Williamson, C.; Craven, J. S.; Duplissy, J.; Adamov, A.; Almeida, J.; Bernhammer, A. K.; Breitenlechner, M.; Brilke, S.; Dias, A.; Ehrhart, S.; Flagan, R. C.; Franchin, A.; Fuchs, C.; Guida, R.; Gysel, M.; Hansel, A.; Hoyle, C. R.; Jokinen, T.; Junninen, H.; Kangasluoma, J.; Keskinen, H.; Kim, J.; Krapf, M.; Kurten, A.; Laaksonen, A.; Lawler, M.; Leiminger, M.; Mathot, S.; Mohler, O.; Nieminen, T.; Onnela, A.; Petaja, T.; Piel, F. M.; Miettinen, P.; Rissanen, M. P.; Rondo, L.; Sarnela, N.; Schobesberger, S.; Sengupta, K.; Sipila, M.; Smith, J. N.; Steiner, G.; Tome, A.; Virtanen, A.; Wagner, A. C.; Weingartner, E.; Wimmer, D.; Winkler, P. M.; Ye, P.; Carslaw, K. S.; Curtius, J.; Dommen, J.; Kirkby, J.; Kulmala, M.; Riipinen, I.; Worsnop, D. R.; Donahue, N. M.; Baltensperger, U. Nature 2016, 533, 527.

[34] Donahue, N. M.; Henry, K. M.; Mentel, T. F.; Kiendler-Scharr, A.; Spindler, C.; Bohn, B.; Brauers, T.; Dorn, H. P.; Fuchs, H.; Tillmann, R.; Wahner, A.; Saathoff, H.; Naumann, K.-H.; Möhler, O.; Leisner, T.; Müller, L.; Reinnig, M.-C.; Hoffmann, T.; Salo, K.; Hallquist, M.; Frosch, M.; Bilde, M.; Tritscher, T.; Barmet, P.; Praplan, A. P.; DeCarlo, P. F.; Dommen, J.; Prévôt, A. S. H.; Baltensperger, U. Proc. Natl. Acad. Sci. U. S. A. 2012, 109, 13503.

[35] Hennigan, C. J.; Miracolo, M. A.; Engelhart, G. J.; May, A. A.; Presto, A. A.; Lee, T.; Sullivan, A. P.; McMeeking, G. R.; Coe, H.; Wold, C. E.; Hao, W. M.; Gilman, J. B.; Kuster, W. C.; de Gouw, J.; Schichtel, B. A.; Collett, J. L.; Kreidenweis, S. M.; Robinson, A. L. Atmos. Chem. Phys. 2011, 11, 7669.

[36] Zhao, B.; Wang, S.; Donahue, N. M.; Chuang, W.; Hildebrandt Ruiz, L.; Ng, N. L.; Wang, Y.; Hao, J. Environ. Sci. Technol. 2015, 49, 2245 .

[37] Müller, L.; Reinnig, M. C.; Naumann, K. H.; Saathoff, H.; Mentel, T. F.; Donahue, N. M.; Hoffmann, T. Atmos. Chem. Phys. 2012, 12, 1483.

[38] Emanuelsson, E. U.; Mentel, T. F.; Watne, A. K.; Spindler, C.; Bohn, B.; Brauers, T.; Dorn, H. P.; Hallquist, A. M.; Haseler, R.; Kiendler-Scharr, A.; Muller, K. P.; Pleijel, H.; Rohrer, F.; Rubach, F.; Schlosser, E.; Tillmann, R.; Hallquist, M. Environ. Sci. Technol. 2014, 48, 6168.

[39] Zhang, X.; Cappa, C. D.; Jathar, S. H.; McVay, R. C.; Ensberg, J. J.; Kleeman, M. J.; Seinfeld, J. H. Proc. Natl. Acad. Sci. U. S. A. 2014, 111,5802 .

[40] Heringa, M. F.; DeCarlo, P. F.; Chirico, R.; Tritscher, T.; Dommen, J.; Weingartner, E.; Richter, R.; Wehrle, G.; Prévôt, A. S. H.; Baltensperger, U. Atmos. Chem. Phys. 2011, 11, 5945.

[41] Bloss, C.; Wagner, V.; Bonzanini, A.; Jenkin, M. E.; Wirtz, K.; Martin-Reviejo, M.; Pilling, M. J. Atmos. Chem. Phys. 2005, 5, 623.

[42] Peng, J. F.; Hu, M.; Du, Z. F.; Wang, Y. H.; Zheng, J.; Zhang, W. B.; Yang, Y. D.; Qin, Y. H.; Zheng, R.; Xiao, Y.; Wu, Y. S.; Lu, S. H.; Wu, Z. J.; Guo, S.; Mao, H. J.; Shuai, S. J. Atmos. Chem. Phys. 2017, 17, 10743 .

[43] Du, Z.; Hu, M.; Peng, J.; Zhang, W.; Zheng, J.; Gu, F.; Qin, Y.; Yang, Y.; Li, M.; Wu, Y.; Shao, M.; Shuai, S. Atmos. Chem. Phys. 2018, 18, 9011.

[44] Mothes, F.; Böge, O.; Herrmann, H. Environ. Sci. Pollut. Res. 2016, 23,15250

[45] Tkacik, D. S.; Presto, A. A.; Donahue, N. M.; Robinson, A. L. Environ. Sci. Technol. 2012, 46, 8773 .

[46] Lambe, A. T.; Onasch, T. B.; Croasdale, D. R.; Wright, J. P.; Martin,
A. T.; Franklin, J. P.; Massoli, P.; Kroll, J. H.; Canagaratna, M. R.; Brune, W. H.; Worsnop, D. R.; Davidovits, P. Environ. Sci. Technol. 2012, 46, 5430 .

[47] Kroll, J. H.; Seinfeld, J. H. Atmos. Environ. 2008, 42, 3593.

[48] Presto, A. A.; Huff Hartz, K. E.; Donahue, N. M. Environ. Sci. Technol. 2005, 39, 7046.

[49] Hoyle, C. R.; Boy, M.; Donahue, N. M.; Fry, J. L.; Glasius, M.; Guenther, A.; Hallar, A. G.; Huff Hartz, K.; Petters, M. D.; Petäjä, T.; Rosenoern, T.; Sullivan, A. P. Atmos. Chem. Phys. 2011, 11, 321.

[50] Surratt, J. D.; Chan, A. W.; Eddingsaas, N. C.; Chan, M.; Loza, C. L.; Kwan, A. J.; Hersey, S. P.; Flagan, R. C.; Wennberg, P. O.; Seinfeld, J. H. Proc. Natl. Acad. Sci. U. S. A. 2010, 107, 6640.

[51] Xu, L.; Guo, H.; Boyd, C. M.; Klein, M.; Bougiatioti, A.; Cerully, K. M.; Hite, J. R.; Isaacman-VanWertz, G.; Kreisberg, N. M.; Knote, C.; Olson, K.; Koss, A.; Goldstein, A. H.; Hering, S. V.; Gouw, J. d.; Baumann, K.; Lee, S.-H.; Nenes, A.; Weber, R. J.; Ng, N. L. Proc. Natl. Acad. Sci. U. S. A. 2015, 112, 37.

[52] Ng, N. L.; Chhabra, P. S.; Chan, A. W. H.; Surratt, J. D.; Kroll, J. H.; Kwan, A. J.; McCabe, D. C.; Wennberg, P. O.; Sorooshian, A.; Murphy, S. M.; Dalleska, N. F.; Flagan, R. C.; Seinfeld, J. H. Atmos. Chem. Phys. 2007, 7, 5159.

[53] Dommen, J.; Metzger, A.; Duplissy, J.; Kalberer, M.; Alfarra, M. R.; Gascho, A.; Weingartner, E.; Prevot, A. S. H.; Verheggen, B.; Baltensperger, U. Geophys. Res. Lett. 2006, 33, L13805.

[54] Chan, A. W. H.; Chan, M. N.; Surratt, J. D.; Chhabra, P. S.; Loza, C. L.; Crounse, J. D.; Yee, L. D.; Flagan, R. C.; Wennberg, P. O.; Seinfeld, J. H. Atmos. Chem. Phys. 2010, 10, 7169.

[55] Zhang, J.; Huff Hartz, K. E.; Pandis, S. N.; Donahue, N. M. J. Phys. Chem. A 2006, 110, 11053.

[56] Zhao, Y.; Saleh, R.; Saliba, G.; Presto, A. A.; Gordon, T. D.; Drozd, G. T.; Goldstein, A. H.; Donahue, N. M.; Robinson, A. L. Proc. Natl. Acad. Sci. U. S. A. 2017, 114, 6984.

[57] Koop, T.; Bookhold, J.; Shiraiwa, M.; Pöschl, U. Phys. Chem. Chem. Phys. 2011, 13, 19238.

[58] Abbatt, J. P.; Lee, A. K.; Thornton, J. A. Chem. Soc. Rev. 2012, 41, 6555.

[59] Zhao, Z.; Xu, Q.; Yang, X.; Zhang, H. ACS Earth Space Chem. 2019, 3, 344 .

[60] Tillmann, R.; Hallquist, M.; Jonsson, Å. M.; Kiendler-Scharr, A.; Saathoff, H.; Iinuma, Y.; Mentel, T. F. Atmos. Chem. Phys. 2010, 10, 7057.

[61] Berkemeier, T.; Steimer, S. S.; Krieger, U. K.; Peter, T.; Poschl, U.; Ammann, M.; Shiraiwa, M. Phys. Chem. Chem. Phys. 2016, 18, 12662.

[62] Chan, M. N.; Zhang, H.; Goldstein, A. H.; Wilson, K. R. J. Phys. Chem. C 2014, 118, 28978.

[63] Li, Z.; Smith, K. A.; Cappa, C. D. Atmos. Chem. Phys. 2018, 18, 14585.

[64] Zhang, X.; McVay, R. C.; Huang, D. D.; Dalleska, N. F.; Aumont, B.; Flagan, R. C.; Seinfeld, J. H. Proc. Natl. Acad. Sci. U. S. A. $\mathbf{2 0 1 5}, 112,14168$.

[65] Kroll, J. H.; Lim, C. Y.; Kessler, S. H.; Wilson, K. R. J. Phys. Chem. A 2015, 119, 10767.

[66] Denisov, E. T.; Afanas'ev, I. B. Oxidation and Antioxidants in Organic Chemistry and Biology, Taylor \& Francis, Boca Roton, 2005, p. 981.

[67] Odum, J. R.; Hoffmann, T.; Bowman, F.; Collins, D.; Flagan, R. C.; Seinfeld, J. H. Environ. Sci. Technol. 1996, 30, 2580.

[68] Ehn, M.; Thornton, J. A.; Kleist, E.; Sipilä, M.; Junninen, H.; Pullinen, I.; Springer, M.; Rubach, F.; Tillmann, R.; Lee, B.; Lopez-Hilfiker, F.; Andres, S.; Acir, I.-H.; Rissanen, M.; Jokinen, T.; Schobesberger, S.; Kangasluoma, J.; Kontkanen, J.; Nieminen, T.; Kurtén, T.; Nielsen, L. B.; Jørgensen, S.; Kjaergaard, H. G.; Canagaratna, M.; Maso, M. D.; Berndt, T.; Petäjä, T.; Wahner, A.; Kerminen, V.-M.; Kulmala, M.; Worsnop, D. R.; Wildt, J.; Mentel, T. F. Nature 2014, 506, 476.

[69] Hu, M.; Shang, D.; Guo, S.; Wu, Z. Acta Chim. Sinica 2016, 74 385. (胡敏, 尚冬杰, 郭松, 吴志军, 化学学报, 2016, 74, 385.)

[70] Seinfeld, J. H.; Pandis, S. N. Atmospheric Chemistry and Physics: From Air Pollution to Climate Change, John Wiley \& Sons, Hoboken, New Jersey, 2006, pp. 464 484.

[71] Wang, Z.; Hu, M.; Wu, Z.; Yue, D. Acta Chim. Sinica 2013, 71, 519. (王志涁, 胡敏, 吴志军, 岳玎利, 化学学报, 2013, 71, 519.)

[72] Eddingsaas, N. C.; VanderVelde, D. G.; Wennberg, P. O. J. Phys. Chem. A 2010, 114, 8106

[73] Minerath, E. C.; Elrod, M. J. Environ. Sci. Technol. 2009, 43, 1386. 
[74] Guo, S.; Hu, M.; Zamora, M. L.; Peng, J.; Shang, D.; Zheng, J.; Du, Z.; Wu, Z.; Shao, M.; Zeng, L.; Molina, M. J.; Zhang, R. Proc. Natl. Acad. Sci. U. S. A. 2014, 111, 17373.

[75] Nozière, B.; Dziedzic, P.; Córdova, A. Geophys. Res. Lett. 2007, 34, L21812.

[76] Nozière, B.; Dziedzic, P.; Córdova, A. Phys. Chem. Chem. Phys. $2010,12,3864$.

[77] Nozière, B.; Córdova, A.; Naturvetenskapliga, F.; Stockholms, U.; Meteorologiska, I. J. Phys. Chem. A 2008, 112, 2827.

[78] Nozière, B.; Dziedzic, P.; Córdova, A.; Naturvetenskapliga, F. J. Phys. Chem. A 2009, 113, 231.

[79] Lee, A. K.; Zhao, R.; Li, R.; Liggio, J.; Li, S. M.; Abbatt, J. P. Environ. Sci. Technol. 2013, 47, 12819.

[80] Kampf, C. J.; Jakob, R.; Hoffmann, T. Atmos. Chem. Phys. 2012, 12, 6323.

[81] Galloway, M. M.; Chhabra, P. S.; Chan, A. W. H.; Surratt, J. D.; Flagan, R. C.; Seinfeld, J. H.; Keutsch, F. N. Atmos. Chem. Phys. 2009, 9, 3331 .

[82] Wang, Z.; Chen, C.; Ma, W.; Zhao, J. J. Phys. Chem. Lett. 2012, 3, 2044.

[83] Tofan-Lazar, J.; Al-Abadleh, H. A. Environ. Sci. Technol. 2014, 48, 394.

[84] Jin, L.; Zhang, P.; Shao, T.; Zhao, S. J. Hazard. Mater. 2014, 271, 9.

[85] Liu, D.; Xiu, Z.; Liu, F.; Wu, G.; Adamson, D.; Newell, C.; Vikesland, P.; Tsai, A. L.; Alvarez, P. J. J. Hazard. Mater. 2013, 262, 456.

[86] Weller, C.; Horn, S.; Herrmann, H. J. Photoch. Photobio. A: Chem. 2013, 255, 41.

[87] Weller, C.; Horn, S.; Herrmann, H. J. Photoch. Photobio. A: Chem. 2013, 268, 24

[88] Willey, D.; Whitehead, R. F.; Kieber, R. J.; Hardison, D. R. Environ. Sci. Technol. 2005, 39, 2579.

[89] Rabani, J.; Klug-Roth, D.; Lilie, J. J. Phys. Chem. 1973, 77, 1169.

[90] Cooper, P. L.; Abbatt, J. P. D. J. Phys. Chem. 1996, 100, 2249.

[91] De Haan, D. O.; Tapavicza, E.; Riva, M.; Cui, T.; Surratt, J. D.; Smith, A. C.; Jordan, M. C.; Nilakantan, S.; Almodovar, M.; Stewart, T. N.; de Loera, A.; De Haan, A. C.; Cazaunau, M.; Gratien, A.; Pangui, E.; Doussin, J. F. Environ. Sci. Technol. 2018, 52, 4061.

[92] Mao, J.; Fan, S.; Jacob, D. J.; Travis, K. R. Atmos. Chem. Phys. 2013, 13, 509 .

[93] Liang, H.; Chen, Z. M.; Huang, D.; Zhao, Y.; Li, Z. Y. Atmos. Chem. Phys. 2013, 13, 11259.

[94] Park, J.; Jang, M.; Yu, Z. Environ. Sci. Technol. 2017, 51, 9605.

[95] Park, J. Y.; Jang, M. RSC Adv. 2016, 6, 58617.

[96] Gustafsson, R. J.; Orlov, A.; Badger, C. L.; Griffiths, P. T.; Cox, R. A.; Lambert, R. M. Atmos. Chem. Phys. 2005, 5, 3415.

[97] Ibrahim, S.; Romanias, M. N.; Alleman, L. Y.; Zeineddine, M. N.; Angeli, G. K.; Trikalitis, P. N.; Thevenet, F. ACS Earth Space Chem. 2018, 2, 376.

[98] Yu, Z.; Jang, M. Atmos. Chem. Phys. 2018, 18, 14609.

[99] Tang, M.; Cziczo, D. J.; Grassian, V. H. Chem. Rev. 2016, 116, 4205.

[100] Yu, Z.; Jang, M. ACS Earth Space Chem. 2019, 3, 2404.

[101] Presto, A. A.; Donahue, N. M. Environ. Sci. Technol. 2006, 40, 3536.

[102] Donahue, N. M.; Robinson, A. L.; Stanier, C.; Pandis, S. N. Environ. Sci. Technol. 2006, 40, 2635.

[103] La, Y. S.; Camredon, M.; Ziemann, P. J.; Valorso, R.; Matsunaga, A.; Lannuque, V.; Lee-Taylor, J.; Hodzic, A.; Madronich, S.; Aumont, B. Atmos. Chem. Phys. 2016, 16, 1417.

[104] Guo, S.; Hu, M.; Peng, J.; Wu, Z.; Zamora, M. L.; Shang, D.; Du, Z.; Zheng, J.; Fang, X.; Tang, R.; Wu, Y.; Zeng, L.; Shuai, S.; Zhang, W.; Wang, Y.; Ji, Y.; Li, Y.; Zhang, A. L.; Wang, W.; Zhang, F.; Zhao, J.; Gong, X.; Wang, C.; Molina, M. J.; Zhang, R. Proc. Natl. Acad. Sci. U.S. A. 2020, 117, 3427.

[105] Peng, J.; Hu, M.; Guo, S.; Du, Z.; Zheng, J.; Shang, D.; Zamora, M. L.; Zeng, L.; Shao, M.; Wu, Y.-S.; Zheng, J.; Wang, Y.; Glen, C. R.; Collins, D. R.; Molina, M. J.; Zhang, R. Proc. Natl. Acad. Sci. U. S. A. 2016, 113,4266

[106] Tkacik, D. S.; Lambe, A. T.; Jathar, S.; Li, X.; Presto, A. A.; Zhao, Y.; Blake, D.; Meinardi, S.; Jayne, J. T.; Croteau, P. L.; Robinson, A. L. Environ. Sci. Technol. 2014, 48, 11235.

[107] Timonen, H.; Karjalainen, P.; Saukko, E.; Saarikoski, S.; Aakko-Saksa, P.; Simonen, P.; Murtonen, T.; Dal Maso, M.; Kuuluvainen, H.; Bloss, M.; Ahlberg, E.; Svenningsson, B.; Pagels, J.; Brune, W. H.; Keskinen, J.; Worsnop, D. R.; Hillamo, R.; Rönkkö, T. Atmos. Chem. Phys. 2017, 17, 5311.

[108] Zhao, Y.; Lambe, A. T.; Saleh, R.; Saliba, G.; Robinson, A. L. Environ. Sci. Technol. 2018, 52, 1253.
[109] Zhang, Y.; Deng, W.; Hu, Q.; Wu, Z.; Yang, W.; Zhang, H.; Wang, Z.; Fang, Z.; Zhu, M.; Li, S.; Song, W.; Ding, X.; Wang, X. Sci. Total Environ. 2020, 722, 137934.

[110] Nordin, E. Z.; Eriksson, A. C.; Roldin, P.; Nilsson, P. T.; Carlsson, J. E.; Kajos, M. K.; Hellén, H.; Wittbom, C.; Rissler, J.; Löndahl, J.; Swietlicki, E.; Svenningsson, B.; Bohgard, M.; Kulmala, M.; Hallquist, M.; Pagels, J. H. Atmos. Chem. Phys. 2013, 13, 6101.

[111] Platt, S. M.; El Haddad, I.; Zardini, A. A.; Clairotte, M.; Astorga, C.; Wolf, R.; Slowik, J. G.; Temime-Roussel, B.; Marchand, N.; Ježek, I.; Drinovec, L.; Močnik, G.; Möhler, O.; Richter, R.; Barmet, P.; Bianchi, F.; Baltensperger, U.; Prévôt, A. S. H. Atmos. Chem. Phys. 2013, 13, 9141 .

[112] Liu, T.; Wang, X.; Deng, W.; Hu, Q.; Ding, X.; Zhang, Y.; He, Q.; Zhang, Z.; Lü, S.; Bi, X.; Chen, J.; Yu, J. Atmos. Chem. Phys. 2015 , $15,9049$.

[113] Liu, T.; Zhou, L.; Liu, Q.; Lee, B. P.; Yao, D.; Lu, H.; Lyu, X.; Guo, H.; Chan, C. K. Environ. Sci. Technol. 2019, 53, 3001.

[114] Jathar, S. H.; Friedman, B.; Galang, A. A.; Link, M. F.; Brophy, P.; Volckens, J.; Eluri, S.; Farmer, D. K. Environ. Sci. Technol. 2017, $51,1377$.

[115] Watne, A. K.; Psichoudaki, M.; Ljungstrom, E.; Le Breton, M.; Hallquist, M.; Jerksjo, M.; Fallgren, H.; Jutterstrom, S.; Hallquist, A. M. Environ. Sci. Technol. 2018, 52, 7720.

[116] Collier, S.; Zhou, S.; Onasch, T. B.; Jaffe, D. A.; Kleinman, L.; Sedlacek, A. J., 3rd; Briggs, N. L.; Hee, J.; Fortner, E.; Shilling, J. E.; Worsnop, D.; Yokelson, R. J.; Parworth, C.; Ge, X.; Xu, J.; Butterfield, Z.; Chand, D.; Dubey, M. K.; Pekour, M. S.; Springston, S.; Zhang, Q. Environ. Sci. Technol. 2016, 50, 8613.

[117] Ortega, A. M.; Day, D. A.; Cubison, M. J.; Brune, W. H.; Bon, D.; de Gouw, J. A.; Jimenez, J. L. Atmos. Chem. Phys. 2013, 13, 11551

[118] Fang, Z.; Deng, W.; Zhang, Y.; Ding, X.; Tang, M.; Liu, T.; Hu, Q.; Zhu, M.; Wang, Z.; Yang, W.; Huang, Z.; Song, W.; Bi, X.; Chen, J.; Sun, Y.; George, C.; Wang, X. Atmos. Chem. Phys. 2017, 17, 14821.

[119] Sanchis, E.; Ferrer, M.; Calvet, S.; Coscollà, C.; Yusà, V.; Cambra-López, M. Atmos. Environ. 2014, 98, 25.

[120] Czech, H.; Pieber, S. M.; Tiitta, P.; Sippula, O.; Kortelainen, M.; Lamberg, H.; Grigonyte, J.; Streibel, T.; Prevot, A. S. H.; Jokiniemi, J.; Zimmermann, R. Atmos. Environ. 2017, 158, 236.

[121] Li, X.; Wang, S.; Duan, L.; Hao, J.; Li, C.; Chen, Y.; Yang, L. Environ. Sci. Technol. 2007, 41, 6052.

[122] Tkacik, D. S.; Robinson, E. S.; Ahern, A.; Saleh, R.; Stockwell, C.; Veres, P.; Simpson, I. J.; Meinardi, S.; Blake, D. R.; Yokelson, R. J.; Presto, A. A.; Sullivan, R. C.; Donahue, N. M.; Robinson, A. L. J. Geophys. Res.: Atmospheres 2017, 122, 6043.

[123] Li, R.; Palm, B. B.; Borbon, A.; Graus, M.; Warneke, C.; Ortega, A. M.; Day, D. A.; Brune, W. H.; Jimenez, J. L.; de Gouw, J. A. Environ. Sci. Technol. 2013, 47, 12566.

[124] Alanen, J.; Simonen, P.; Saarikoski, S.; Timonen, H.; Kangasniemi, O.; Saukko, E.; Hillamo, R.; Lehtoranta, K.; Murtonen, T.; Vesala, H.; Keskinen, J.; Ronkko, T. Atmos. Chem. Phys. 2017, 17, 8739.

[125] Liu, T.; Li, Z.; Chan, M.; Chan, C. K. Atmos. Chem. Phys. 2017, 17, 7333.

[126] Saha, P. K.; Reece, S. M.; Grieshop, A. P. Environ. Sci. Technol. 2018, 52, 7192 .

[127] Liu, J.; Chu, B.; Chen, T.; Liu, C.; Wang, L.; Bao, X.; He, H. Environ. Sci. Technol. 2018, 52, 6834.

[128] Palm, B. B.; de Sa, S. S.; Day, D. A.; Campuzano-Jost, P.; Hu, W. W.; Seco, R.; Sjostedt, S. J.; Park, J. H.; Guenther, A. B.; Kim, S.; Brito, J.; Wurm, F.; Artaxo, P.; Thalman, R.; Wang, J.; Yee, L. D.; Wernis, R.; Isaacman-VanWertz, G.; Goldstein, A. H.; Liu, Y. J.; Springston, S. R.; Souza, R.; Newburn, M. K.; Alexander, M. L.; Martin, S. T.; Jimenez, J. L. Atmos. Chem. Phys. 2018, 18, 467.

[129] Kang, E.; Lee, M.; Brune, W. H.; Lee, T.; Park, T.; Ahn, J.; Shang, X. Atmos. Chem. Phys. 2018, 18, 6661.

[130] Canagaratna, M. R.; Jimenez, J. L.; Kroll, J. H.; Chen, Q.; Kessler, S. H.; Massoli, P.; Hildebrandt Ruiz, L.; Fortner, E.; Williams, L. R.; Wilson, K. R.; Surratt, J. D.; Donahue, N. M.; Jayne, J. T.; Worsnop, D. R. Atmos. Chem. Phys. 2015, 15, 253.

[131] Li, K.; Liggio, J.; Lee, P.; Han, C.; Liu, Q.; Li, S.-M. Atmos. Chem. Phys. 2019, 19, 9715.

[132] Holzinger, R.; Williams, J.; Herrmann, F.; Lelieveld, J.; Donahue, N. M.; Roeckmann, T. Atmos. Chem. Phys. 2010, 10, 2257.

[133] Saleh, R.; Hennigan, C. J.; McMeeking, G. R.; Chuang, W. K.; Robinson, E. S.; Coe, H.; Donahue, N. M.; Robinson, A. L. Atmos. Chem. Phys. 2013, 13, 7683.

(Cheng, B.; Fan, Y.) 